\title{
Parasympathetic dysfunction and antiarrhythmic effect of vagal nerve stimulation following myocardial infarction
}

\author{
Marmar Vaseghi,, ${ }^{1,2,3}$ Siamak Salavatian, ${ }^{1,2,3}$ Pradeep S. Rajendran, ${ }^{1,2,3}$ Daigo Yagishita, ${ }^{1,2}$ \\ William R. Woodward, ${ }^{4}$ David Hamon, ${ }^{1,2}$ Kentaro Yamakawa, ${ }^{2}$ Tadanobu Irie, ${ }^{1,2}$ Beth A. Habecker, ${ }^{4,5}$ \\ and Kalyanam Shivkumar ${ }^{1,2,3}$ \\ ${ }^{1}$ Cardiac Arrhythmia Center, ${ }^{2}$ Neurocardiology Research Center of Excellence, and ${ }^{3}$ Molecular Cellular and Integrative \\ Physiology Interdepartmental Program, UCLA, Los Angeles, California, USA. ${ }^{4}$ Department of Physiology \& Pharmacology \\ and ${ }^{5}$ Department of Medicine Knight Cardiovascular Institute, Oregon Health and Science University, \\ Portland, Oregon, USA.
}

Myocardial infarction causes sympathetic activation and parasympathetic dysfunction, which increase risk of sudden death due to ventricular arrhythmias. Mechanisms underlying parasympathetic dysfunction are unclear. The aim of this study was to delineate consequences of myocardial infarction on parasympathetic myocardial neurotransmitter levels and the function of parasympathetic cardiac ganglia neurons, and to assess electrophysiological effects of vagal nerve stimulation on ventricular arrhythmias in a chronic porcine infarct model. While norepinephrine levels decreased, cardiac acetylcholine levels remained preserved in border zones and viable myocardium of infarcted hearts. In vivo neuronal recordings demonstrated abnormalities in firing frequency of parasympathetic neurons of infarcted animals. Neurons that were activated by parasympathetic stimulation had low basal firing frequency, while neurons that were suppressed by left vagal nerve stimulation had abnormally high basal activity. Myocardial infarction increased sympathetic inputs to parasympathetic convergent neurons. However, the underlying parasympathetic cardiac neuronal network remained intact. Augmenting parasympathetic drive with vagal nerve stimulation reduced ventricular arrhythmia inducibility by decreasing ventricular excitability and heterogeneity of repolarization of infarct border zones, an area with known proarrhythmic potential. Preserved acetylcholine levels and intact parasympathetic neuronal pathways can explain the electrical stabilization of infarct border zones with vagal nerve stimulation, providing insight into its antiarrhythmic benefit.

Conflict of interest: The authors have declared that no conflict of interest exists.

Submitted: January 25, 2016

Accepted: July 6, 2017

Published: August 17, 2017

Reference information: JCI Insight. 2017;2(16):e86715. https://doi.org/10.1172/jci. insight.86715.

\section{Introduction}

The autonomic nervous system plays a critical role in the genesis and maintenance of ventricular tachyarrhythmias (VT) $(1,2)$. In particular, sympathetic activation and parasympathetic dysfunction are known to accompany myocardial infarction (MI) and increase the risk of sudden cardiac death $(1,2)$. Parasympathetic dysfunction has been indirectly studied and manifests as abnormal baroreflex sensitivity and heart rate variability in cardiomyopathy patients (3-6). Mechanisms behind these abnormalities - and importantly, the level at which they occur (cardiac vs. extracardiac) - are poorly characterized. Multiple studies have shown that vagal nerve stimulation (VNS) reduces inflammation and ischemia-driven ventricular arrhythmias if initiated at the time of or before onset of coronary artery occlusion (7-10). However, introducing VNS before or even at the onset of ischemia is not clinically feasible. Furthermore, many gaps exist in our knowledge regarding vagal control of cardiac excitability, parasympathetic neural control and remodeling, and alterations in cardiac parasympathetic neurotransmitters with MI. Therefore, it is unknown if VNS can reduce recurrent VT in the setting of a chronic MI when pathological myocardial remodeling with scar formation and neural remodeling has occurred. It is also unknown if VNS can be used acutely for treatment of ventricular tachycardia/ventricular fibrillation (VT/VF) storm in patients with cardiomyopathy. 
Implications of this therapy are critical for the many patients with heart failure who experience recurrent internal, cardiac defibrillator shocks due to VT, despite catheter ablation and medical therapies (11-13).

In this study, we hypothesized that MI causes a decrease in parasympathetic drive from the CNS. As evidence for this, we hypothesized that the primary parasympathetic neurotransmitter acetylcholine (ACh) would remain intact in the setting of MI, but that parasympathetic neurons in the intrinsic cardiac ganglia (on the surface heart) would demonstrate abnormal activity due to a decrease in central drive. Furthermore, we hypothesized that increasing this drive with VNS could acutely restore electrical stability by modulating cardiac excitability and repolarization, a mechanism that, unlike previously described cellular and anti-inflammatory effects of VNS, could work rapidly to reduce arrhythmia burden. We investigated these questions in a porcine infarct model, utilizing neurotransmitter measurements, direct neuronal recordings, and electrophysiological mapping.

\section{Results}

Neurotransmitter analysis. Six to 8 weeks after percutaneous MI (Supplemental Figure 1; supplemental material available online with this article; https://doi.org/10.1172/jci.insight.86715DS1), myocardial $\mathrm{ACh}$ and norepinephrine (NE) content from scar, border zone, and viable regions of infarcted hearts was analyzed, guided by electronatomic bipolar voltage mapping prior to sampling of each of these regions (Figure 1A). In addition, in normal animals, ACh and NE levels across the left ventricular (LV) apex, anterior, and lateral walls were also analyzed and compared with infarcted hearts. There was no significant difference in ACh levels across the LV epicardium, including LV apex, anterior, and lateral walls of normal hearts (Figure 1B). Furthermore, there was no significant difference between ACh content of the epicardium and endocardium of normal animals (Figure 1C). In infarcted hearts, regional ACh analysis demonstrated that scar regions (with voltage $<0.5 \mathrm{mV}$ ) had the lowest $\mathrm{ACh}$ levels as compared with border zone and viable myocardium (Figure 1D, $P \leq 0.01$ ). Although ACh content of scar areas was, to some extent, reduced compared with normal hearts, this difference was not statistically significant $(P=0.1)$. Therefore, cardiac ACh levels in infarcted animals, particularly in border zone and viable myocardium, were unchanged compared with normal animals.

Similar to ACh, NE levels were similar across the LV epicardium (LV apex, LV anterior wall, and LV lateral wall), as well as on the epicardium, compared with the endocardium of normal hearts (Figure 1, E and $\mathrm{F}$ ). However, unlike $\mathrm{ACh}, \mathrm{NE}$ content in scar regions was significantly reduced compared with normal hearts (Figure $1 \mathrm{G}$ ). In infarcted hearts, NE levels of viable myocardium remained relatively normal and greater than border zone regions. Scar demonstrated the lowest NE content (Figure 1G).

MI alters basal activity of parasympathetic neurons. Given that ACh levels in infarcted hearts were maintained, basal activity of parasympathetic neurons of the cardiac ganglia was then evaluated to discern differences in the firing frequency of these neurons between normal and infarcted animals. The ventral interventricular (VIV) fat pad, located below the left atrial appendage in the porcine heart (Supplemental Figure 1), contains multiple ganglia that innervate the ventricular myocardium. In vivo recordings from these neurons were obtained using a multielectrode array. An increase (Figure 2) or decrease (Figure 3) in firing frequency of a recorded neuron in response to right or left VNS was used to classify it as a parasympathetic efferent neuron (14). Basal activity of neurons that increased their firing frequency in response to left VNS was reduced in infarcted compared with normal animals $(0.14 \pm 0.04 \mathrm{~Hz}$ vs. $0.29 \pm 0.07 \mathrm{~Hz}$, respectively, $P$ $<0.05$; Figure 2C). Furthermore, the basal activity of neurons that decrease their firing in response to left VNS was increased in infarcted compared with animals with normal hearts $(0.56 \pm 0.14 \mathrm{~Hz}$ vs. $0.31 \pm 0.05$ $\mathrm{Hz}$, respectively, $P<0.05$; Figure $3 \mathrm{C}$ ). No significant difference in basal activity of neurons that increase or decrease their firing frequency to right VNS was found (Figure 2D and Figure 3D). Therefore, neurons receiving inputs from the left vagal trunk demonstrated abnormal activity at baseline in infarcted compared with normal hearts, suggesting a decrease in central drive through the left vagus.

Cardiac parasympathetic neuronal function. We then further classified parasympathetic neurons in the cardiac ganglia as purely efferent or convergent in order to assess if MI caused remodeling of the type of inputs these neurons received. A parasympathetic neuron (classified by its response to right or left VNS) that also responded to an afferent stimulus, including epicardial mechanosensory stimuli and/or changes in preload and afterload, was further classified as a convergent parasympathetic neuron. Convergent neurons are thought to be responsible for the majority of local cardiac neural processing $(14,15)$. A neuron that did not respond to afferent stimuli was classified as an efferent neuron. In this study, the proportion of 
A

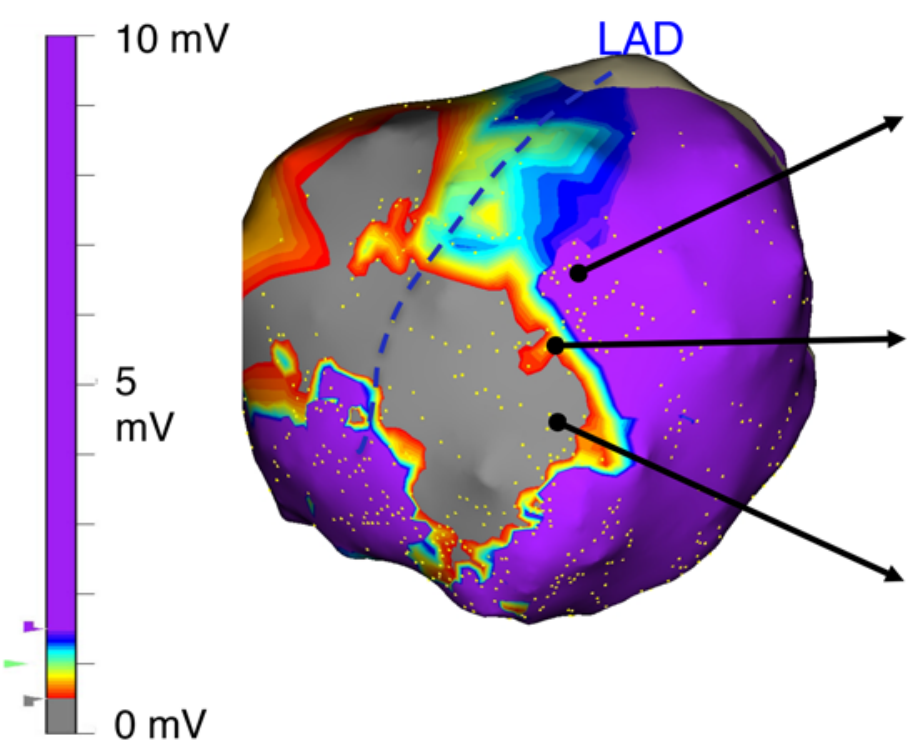

B

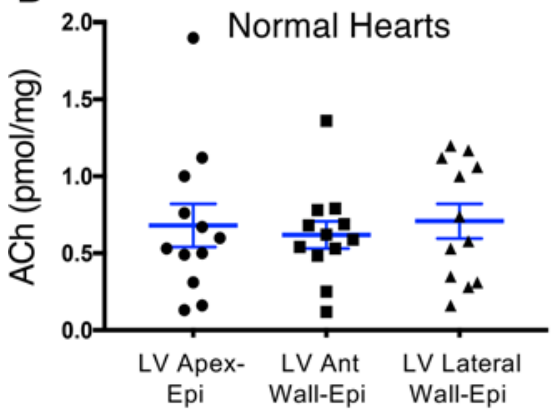

E

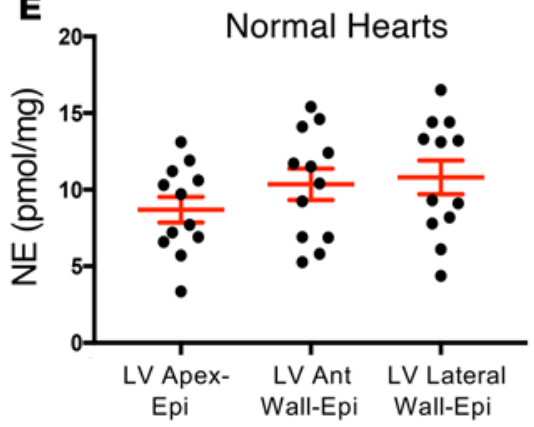

C

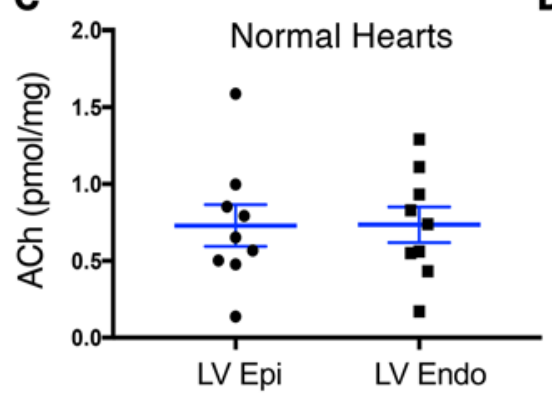

$\mathbf{F}$

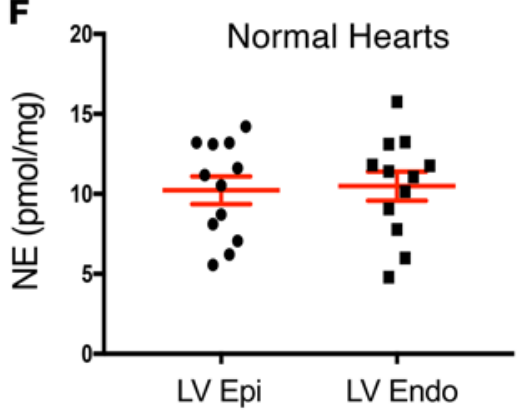

(1) MI-Viable (Bipolar Voltage > $1.5 \mathrm{mV}$ )

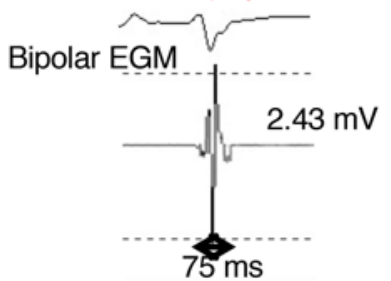

Unipolar EGM

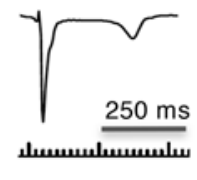

(2)MI-Border zone (Bipolar Voltage 0.5-1.5 mV) Bipolar EGM

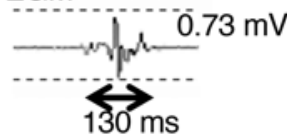

Unipolar EGM

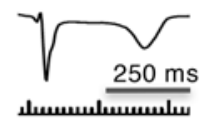

(3) MI-Scar (Bipolar Voltage $<0.5 \mathrm{mV}$ )
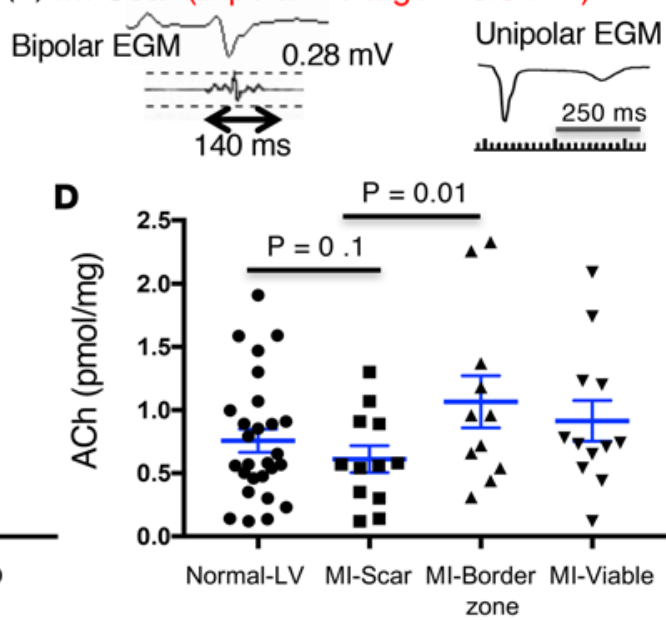

G

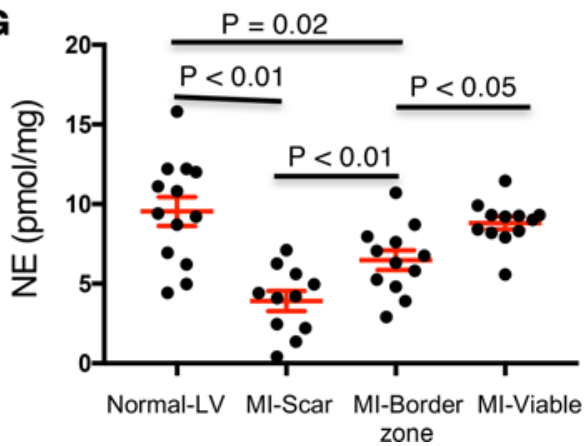

Figure 1. Cardiac voltage mapping and neurotransmitter analysis. (A) Bipolar voltage electroanatomic maps of infarcted hearts were obtained and used to identify viable, border zone, and scar regions. Examples of bipolar electrical recordings from viable, border zone, and scar regions are shown. Examples of uinpolar electrograms obtained from similar regions are also shown. This map and bipolar voltage measurements were used to obtain samples for neurotransmitter analysis from appropriate locations. The scale bar shows the voltage from 0-10 mV. (B) In normal hearts ( $n=12$ normal animals, 3 samples per animal), acetylcholine (ACh) levels were not significantly different on the LV apex, anterior, lateral walls (linear mixed effects model). (C) ACh levels from paired LV epicardium and endocardium samples were also not significantly different ( $n=9$ normal animals, $2-3$ samples per animal). (D) In infarcted hearts $(n=14)$, scar regions have the lowest ACh content compared with viable and border zone regions $(P<0.01$, linear mixed effects model, $1-3$ samples per region per animal). However, ACh content between viable and border zone regions of infarcted animals $(n=14)$ compared with similar regions of normal hearts ( $n=15$ animals) was not statistically significant $(P=0.4$, linear mixed effects model). There is a suggestion that scar ACh may be somewhat reduced compared with normal hearts; however, this difference did not reach statistical significance. (E) Similar to ACh levels, there was no difference in norepinephrine (NE) content across the LV apex, anterior, or lateral wall (linear mixed effects model, $n=12$ normal animals, 3 samples per animal). (F) Also, similar to ACh, NE levels in the endocardium were similar to epicardium (linear mixed effects model, $n=9$ animals, 2-3 samples per animal). (C) Unlike ACh, the NE content in scar and border zone regions was significantly reduced compared with viable myocardium in infarcted hearts $(P<0.05$ for border zone vs. viable, and $P<0.01$ for scar vs. viable, linear mixed effects model, $n=5$ animals, $1-3$ samples per region per animal) and was significantly less than similar regions in normal hearts $(P<0.01$, linear mixed effects model). There was no difference in NE content of viable regions of infarcted hearts compared with normal hearts. MI, myocardial infarction; EGM, myocardial electrogram; LV, left ventricle; Epi, epicardium; Endo, endocardium. 
A

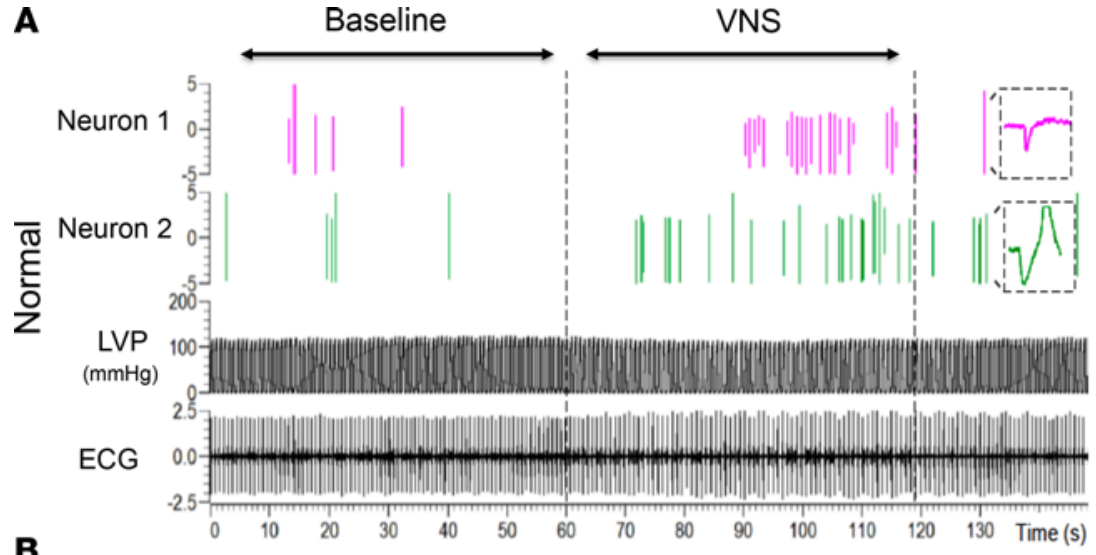

B

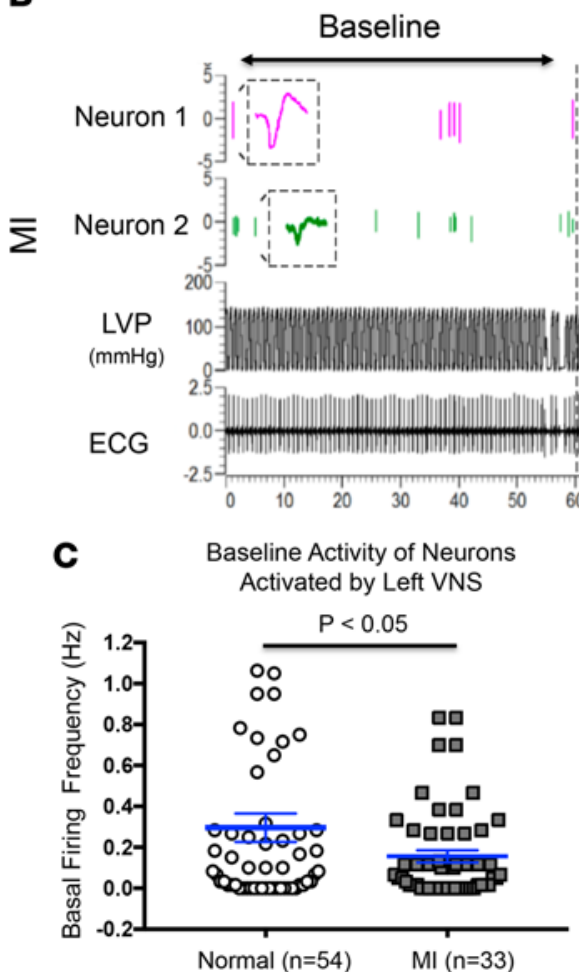

VNS
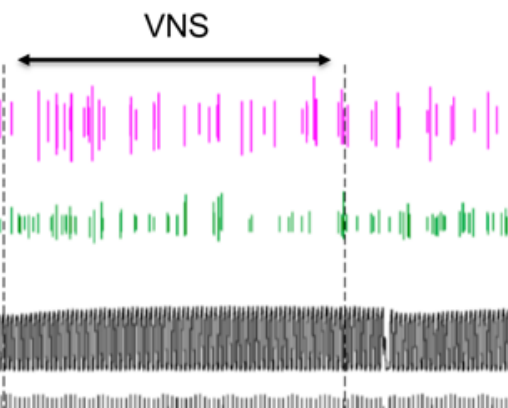

D
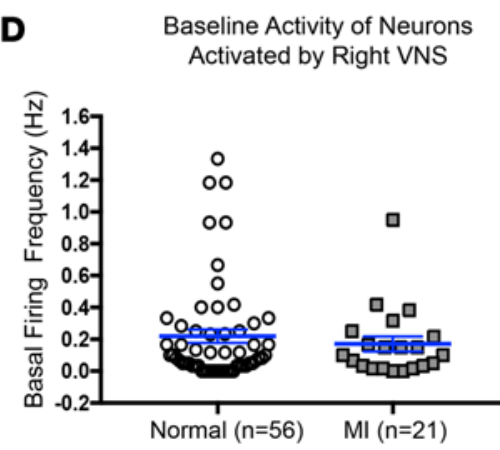

Figure 2. Recordings from cardiac neurons that are activated by VNS. (A) Example of direct neuronal recordings from the ventral interventricular (VIV) fat pad ganglia of a normal heart showing 2 neurons that increased their firing activity with VNS and were therefore classified as postganglionic parasympathetic neurons. Each neuron was also identified and classified by its unique neuronal waveform (inset). (B) Example of neuronal recordings from the VIV fat pad of a heart with myocardial infarction (MI) demonstrating 2 neurons that also increased their firing frequency with VNS. Basal activity in the minute prior to VNS compared with during VNS was used to identify parasympathetic neurons. (C) The basal (prestimulation) activity of parasympathetic neurons that were activated by left VNS is reduced in infarcted hearts compared with normal hearts ( $n=54$ neurons from 15 normal and $n=33$ neurons from 10 infarcted animals, $P<0.05$, unpaired Student's $t$ test). (D) Basal activity of neurons that respond to right VNS is unchanged in normal vs. infarcted hearts ( $n=56$ neurons from 15 normal and $n=33$ neurons from 10 infarcted animals, $P=0.4$, unpaired Student's $t$ test). LVP, left ventricular pressure; VNS, vagal nerve stimulation.

convergent neurons was unchanged in infarcted compared with normal hearts ( $81 \%$ of total neurons responding to left VNS were convergent in normal vs. $79 \%$ in infarcted hearts, $P=0.8 ; 82 \%$ of total neurons responding to right VNS were convergent in normal vs. $82 \%$ in infarcted hearts, $P=0.9$ ). We then evaluated if the amount of sympathetic input received by these convergent neurons was altered due to MI. Therefore, the activity of parasympathetic convergent neurons in response to right or left stellate stimulation was assessed. The proportion of convergent parasympathetic neurons that also received sympathetic input from the right or left stellate ganglion (e.g., responded to right or left stellate ganglion stimulation) was significantly increased in infarcted compared with normal hearts ( $25 \%$ of left vagal convergent neurons received sympathetic input in normal vs. $51 \%$ in infarcted hearts, $P<0.01$; and $25 \%$ of right vagal convergent neurons received sympathetic input in normal vs. $56 \%$ in infarcted hearts, $P<0.01$; Figure $4, \mathrm{~A}$ and B). There was no difference in the proportion of purely efferent parasympathetic neurons that also received sympathetic input in infarcted vs. normal hearts (Figure 4).

Conditional probability analysis demonstrated that the functional connectivity or the primary relationships of parasympathetic neurons in the cardiac ganglia remained intact in infarcted hearts (Figure 4, C and E). However, there was increasing sympathetic input to convergent parasympathetic neurons, particularly those that received right ventricle (RV) sensory afferent input and transduced a decrease in preload (Figure 4, C and E). Despite increased sympathetic neurotransmission and changes in the basal activity of neurons receiving left vagal input, the underlying parasympathetic network at the level of the cardiac ganglia remained intact in infarcted hearts, suggesting that increasing central drive with VNS could still take advantage of these remaining pathways in infarcted hearts to promote electrical stability (Figure 4, D and F).

Electrical stabilization of infarct border zones by bilateral VNS. Electrophysiological effects of VNS can be determined by simultaneous high resolution mapping and measurement of activation recovery intervals (ARI), a surrogate of local action potential duration, from multiple epicardial ventricular unipolar electrodes on a 56-electrode sock (Figure 5A and Supplemental Figure 1) without causing myocardial 

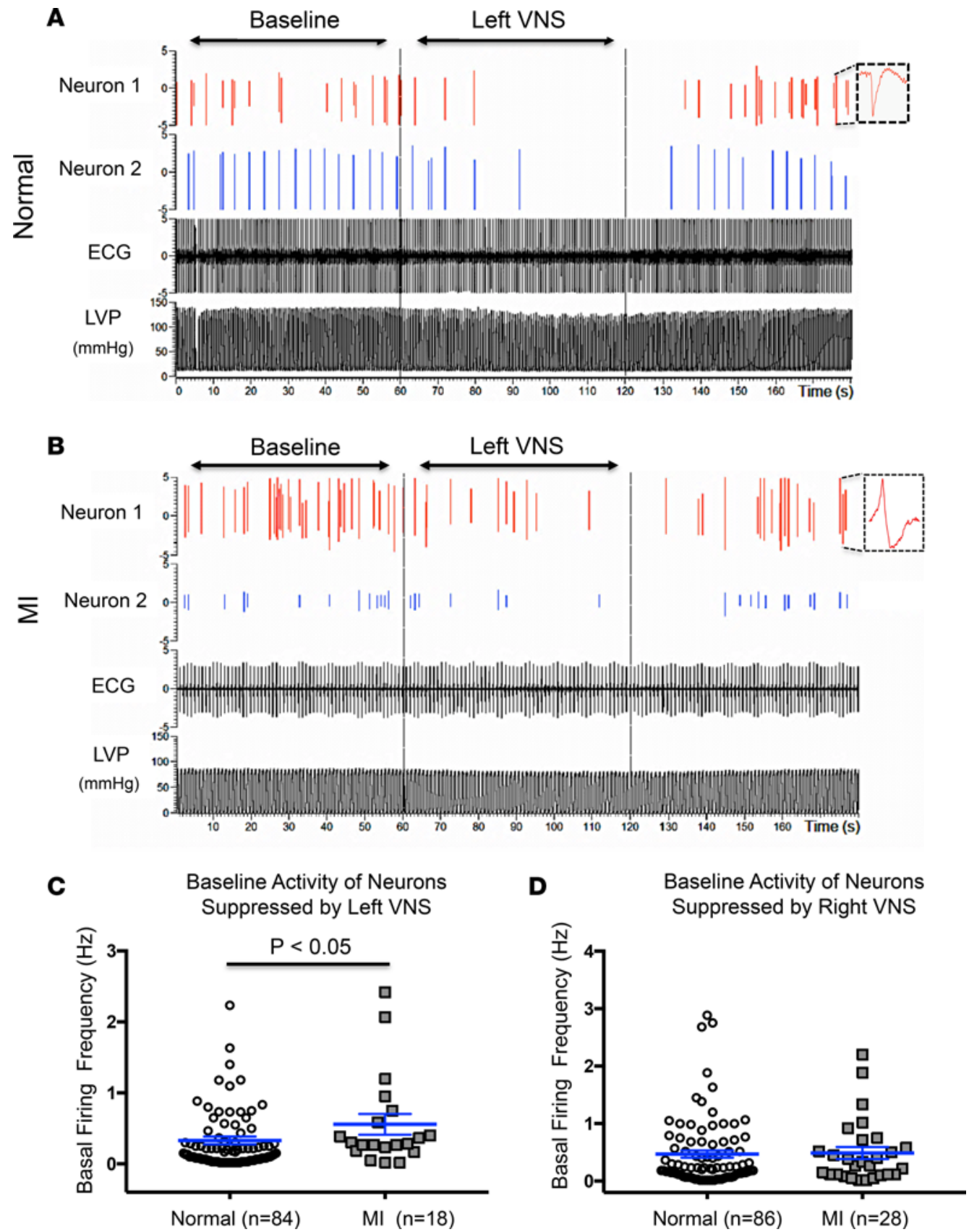

Figure 3. Recordings from cardiac neurons that are suppressed by VNS. (A) Example of direct neuronal recordings from the VIV fat pad ganglia of a normal heart showing 2 neurons that decrease their firing activity with VNS and were therefore classified as postganglionic parasympathetic neurons. Each neuron was identified and classified by its unique neuronal waveform (inset). (B) Example of neuronal recordings from the VIV fat pad of an infarcted heart demonstrating 2 neurons that also decreased their firing frequency with VNS. Basal activity in the minute prior to VNS compared with during VNS was used to identify parasympathetic neurons. (C) The basal (prestimulation) activity of parasympathetic neurons that were suppressed by left VNS is reduced in MI compared with normal hearts $(P<0.05$, unpaired Student's $t$ test, $n=84$ neurons from 15 normal and $n=18$ neurons from infarcted animals). (D) Basal activity of neurons that are suppressed by right VNS is unchanged in normal vs. infarcted hearts $(P=0.4$, unpaired Student's $t$ test, $n=86$ neurons from 15 normal and $n=28$ neurons from 10 infarcted animals). LVP, left ventricular pressure; MI, myocardial infarction; VNS, vagal nerve stimulation.

injury. Given that central parasympathetic drive to the cardiac ganglia appeared to be reduced in infarcted hearts, while the underlying parasympathetic pathways and overall percentage of convergent and efferent neurons were intact, we hypothesized that restoring central drive with VNS could improve electrical stability, particularly in the absence of significant alterations in ACh levels. There was no difference in the stimulation current used in normal and infarcted hearts (right VNS $2.4 \pm 0.3 \mathrm{~mA}$ vs. $2.2 \pm 0.5 \mathrm{~mA}, P=$ 0.6 ; left VNS $2.6 \pm 0.5 \mathrm{~mA}$ vs. $2.3 \pm 0.4 \mathrm{~mA}$, respectively, $P=0.6$ ). Intermittent VNS decreased heart rate 
A

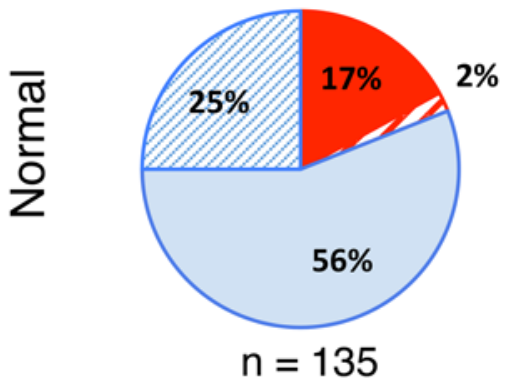

$n=53$

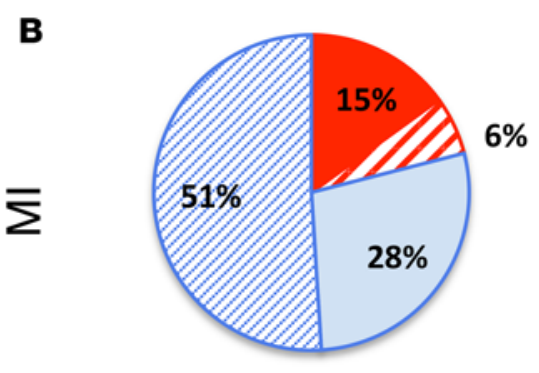

Darasympathetic Eff Only

Parasymapthetic Eff + Symp Input

$\square$ Parasympathetic Convergent Only

$\square$ Parasympathetic Convergent + Symp Input

Parasympathetic Eff only

$\checkmark$ Parasympathetic Eff + Symp Input

$\square$ Parasympathetic Convergent only

$\square$ Parasympathetic Convergent + Symp Input

Control (response to $\mathrm{Y}$ I response to $\mathrm{X}$ )

C
즌
은

$\mathbf{E}$

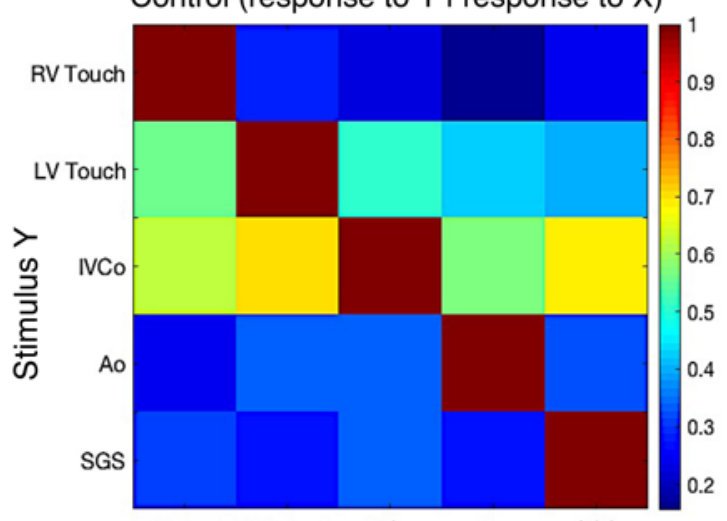

RV Touch LV Touch IVCo Ao SGS

Stimulus $X$

$\mathrm{MI}$ (response to $\mathrm{YI}$ response to $\mathrm{X}$ )

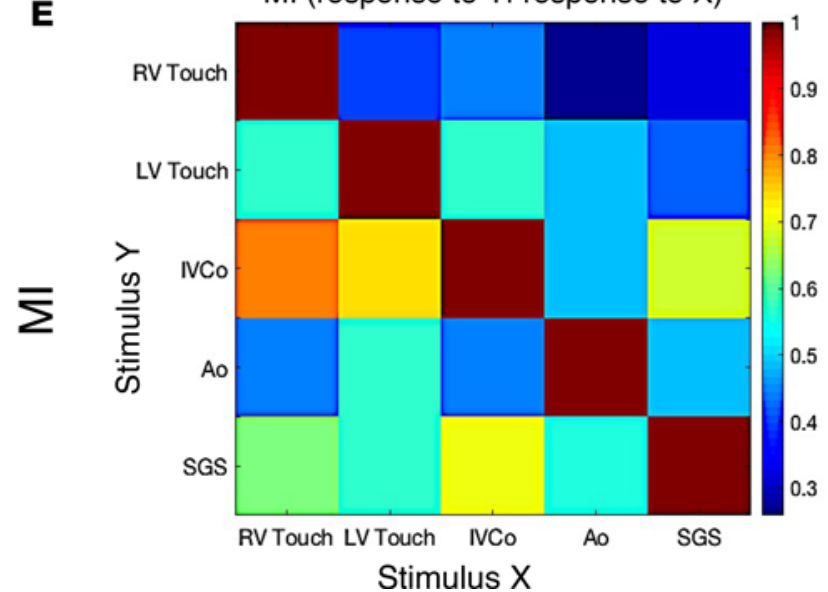

Right Vagus
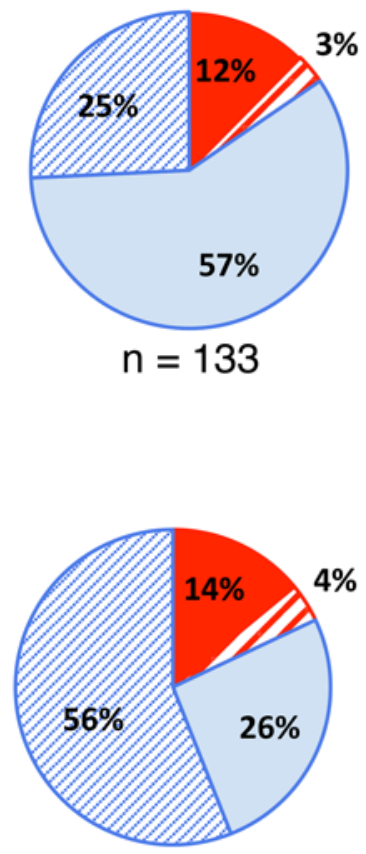

$\mathrm{n}=50$ $n=133$

- Parasympathetic Eff Only

Darasymapthetic Eff + Symp Input

$\square$ Parasympathetic Convergent Only

■ Parasympathetic Convergent + Symp Input

Parasympatheti Eff only

D Parasympathetic Eff + Symp Input

$\square$ Parasympathetic Convergent Only

$\square$ Parasympathetic Convergent + Symp Input

D

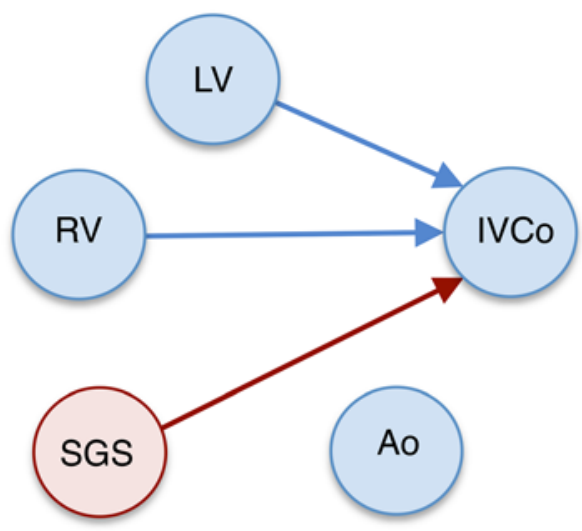

$\mathbf{F}$

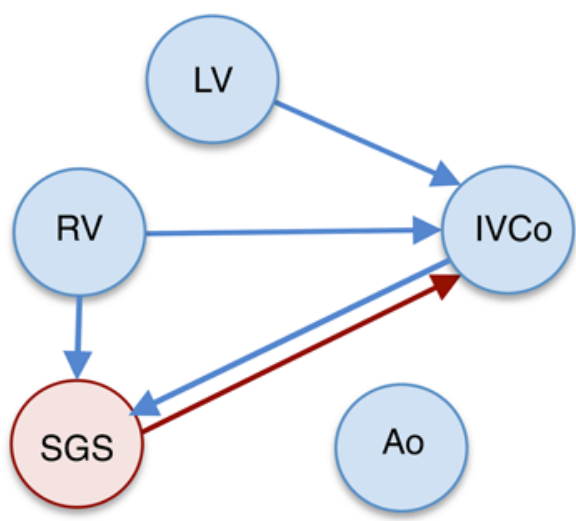


Figure 4. Classification and relationships of parasympathetic neurons in normal and infarcted hearts. (A) Functional classification of parasympathetic neurons that receive inputs from the left or right vagus is shown in normal hearts. Overall, percentage of convergent parasympathetic neurons that responded to left VNS and at least 1 afferent stimulus (therefore classified as convergent) was $81 \%$ (109 of 135 left VNS responsive neurons from 15 normal animals), and the percentage of convergent neurons that responded to right VNS was 82\% (109 of 133 right VNS responsive neurons from 15 normal animals). Of these convergent neurons, approximately $30 \%$ also received sympathetic input in normal hearts. (B) Type of parasympathetic neurons that receive input from the left or right vagus is shown in hearts with myocardial infarction (MI). Percentage of convergent neurons that responded to left VNS was $79 \%$ ( 42 of 53 left VNS responsive neurons from 10 infarcted animals), and percentage of convergent neurons that responded to right VNS was $82 \%$ (41 of 50 total neurons from 10 infarcted animals). These values were not different than normal hearts $\left(P=0.8, \chi^{2}\right.$ test). However, percentage of convergent neurons also receiving sympathetic input was significantly increased in infarcted ( $n=15$ animals) compared with normal hearts ( $n=10$ animals), representing $65 \%$ (27 of 47 convergent neurons) and 68\% (28 of 50 convergent neurons) for left and right VNS in infarcted animals, respectively, compared with 31\% (34 of 109 convergent neurons) and 30\% (33 of 109 convergent neurons) for left and right VNS in normal hearts, respectively $\left(P<0.05, \chi^{2}\right.$ test). (C) Conditional probability analysis (probability that a VNS neuron that responded to one stimulus [X] also responded to another stimulus [Y]) for neurons that respond to VNS is shown in normal hearts. Significant relationships are noted for parasympathetic neurons that transduce preload and sensory inputs from the RV and LV due to touch, as well as those that transduce preload and receive sympathetic input. (D) Graphical representation of the conditional probability analysis is shown for VNS-responsive neurons of normal hearts, where significant relationships (those with a value $\geq 0.6$ ) are delineated. (E) Conditional probability analysis for neurons that respond to VNS in infarcted hearts is shown. Significant relationships are noted for parasympathetic neurons that transduce preload and sensory inputs from the RV and LV due to touch, as well as those convergent neurons that transduce preload and receive sympathetic input. However, additional interactions of convergent neurons with sympathetic ganglion neurons exist in infarcted hearts. (F) Graphical representation of the conditional probability analysis is shown for parasympathetic neurons of infarcted hearts, where significant relationships (those with a value $\geq 0.6$ ) are delineated. Eff, efferent; Symp, sympathetic; RV, right ventricle; LV, left ventricle; IVCo, IVC occlusion; Ao, aortic occlusion; SCS, sympathetic ganglion stimulation; VNS, vagal nerve stimulation; MI, myocardial infarction.

from $86 \pm 3.8 \mathrm{bpm}$ to $71 \pm 2.5 \mathrm{bpm}$, systolic blood pressure from $123 \pm 6.5 \mathrm{mmHg}$ to $113 \pm 7.1 \mathrm{mmHg}$, and diastolic blood pressure from $92 \pm 5 \mathrm{mmHg}$ to $79 \pm 6 \mathrm{mmHg}$ in normal animals $(P<0.001$ for all parameters). Global epicardial ARI obtained from unipolar electrograms (Figure 5A) increased from 336 $\pm 13 \mathrm{~ms}$ to $354 \pm 15 \mathrm{~ms}$ (Figure $5 \mathrm{~B}, 5.4 \% \pm 1 \%, P=0.035$ ) in normal hearts.

In infarcted animals, VNS decreased heart rate from $84 \pm 3 \mathrm{bpm}$ to $70 \pm 3 \mathrm{bpm}$, systolic blood pressure from $112 \pm 6 \mathrm{mmHg}$ to $99 \pm 6 \mathrm{mmHg}$, and diastolic blood pressure from $99 \pm 6 \mathrm{mmHg}$ to $65 \pm 4 \mathrm{mmHg}$ $(P<0.001$ for all parameters compared with baseline). Global epicardial ARI increased from $361 \pm 13 \mathrm{~ms}$ to $405 \pm 12 \mathrm{~ms}(13 \% \pm 2 \%, P<0.001$; Figure $5 \mathrm{C})$. The percentage change in global ARI in infarcted hearts was greater than in normal hearts $(P=0.035)$, despite similar stimulation currents.

In infarcted hearts, the scar regions had the greatest ARI at baseline (397 $\pm 14 \mathrm{~ms})$ as compared with border zone $(380 \pm 14 \mathrm{~ms})$ or viable myocardium $(363 \pm 14 \mathrm{~ms} ; P<0.01$, Figure $5 \mathrm{D})$. VNS significantly increased ARI in all regions (scar by $9.7 \pm 11 \%$, border zones by $14 \pm 3.5 \%$, and viable myocardium by $12 \pm 3 \%, P<0.001$ compared with baseline); however, border zone and viable regions showed a greater increase in ARI than scar regions $(P<0.05$, Figure $5 \mathrm{D})$. Given that scar regions had a higher ARI at baseline, VNS led to an overall reduction in global epicardial heterogeneity in ARIs.

VT inducibility was assessed in 21 infarcted animals by ventricular extrastimulus pacing at baseline and during VNS (Figure 6A). Twelve (57\%) of 21 infarcted animals were inducible for VT that required defibrillation at baseline (Figure 6B). Activation maps demonstrated significant areas of slowed conduction along border zones during VT (Figure 6C). During intermittent VNS, only 5 of 21 (23\%) of infarcted animals were inducible (Figure 6B). There was one animal, however, that was not inducible at baseline and was inducible with triple extrastimuli during VNS. Overall, VNS significantly decreased inducibility of ventricular arrhythmias by $60 \%(P<0.05)$.

Given reduction in VT inducibility, the dispersion in ARI or heterogeneity in repolarization in various regions of the infarcted myocardium was analyzed. At baseline, border zone regions showed the greatest dispersion in ARI $387 \pm 12 \mathrm{~ms}^{2}$ compared with scar $\left(73 \pm 23 \mathrm{~ms}^{2}\right)$ and viable $\left(160 \pm 25 \mathrm{~ms}^{2}\right)$ myocardium (Figure 6D), and they were often sites of VT exit and slowed conduction (Figure 6C). During VNS, ARI dispersion of border zone regions decreased to $185 \pm 63 \mathrm{~ms}^{2}(P<0.05$; Figure 6E). Scar regions did not show a significant change in dispersion during VNS (from $73 \pm 23 \mathrm{~ms}^{2}$ to $69 \pm 26$ $\mathrm{ms}^{2}$ ), while there was suggestion of a mild increase in dispersion in viable regions from $160 \pm 25 \mathrm{~ms}^{2}$ to $253 \pm 188 \mathrm{~ms}^{2}(P=0.1 ;$ Figure $6 \mathrm{E})$.

Delineation of scar: electroanatomic mapping, cardiac MRI, and histology. In all infarcted hearts, epicardial scar location on cardiac MRI was similar to low-voltage regions delineated by electroanatomic mapping (Figure 7). High-resolution cardiac MRI demonstrated areas of normal myocardium within regions of delayed enhancement that had recorded a voltage less than $0.5 \mathrm{mV}$ (Figure 7), indicating that scar regions contained islands of viable myocardium, a finding that has been confirmed in patients 
A

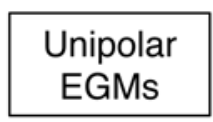

Normal LV

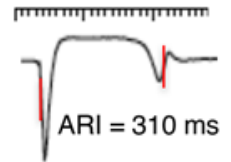

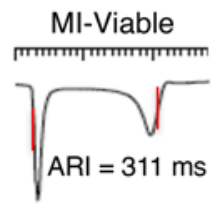
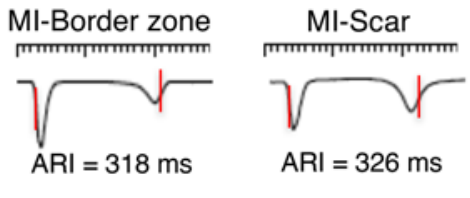

B

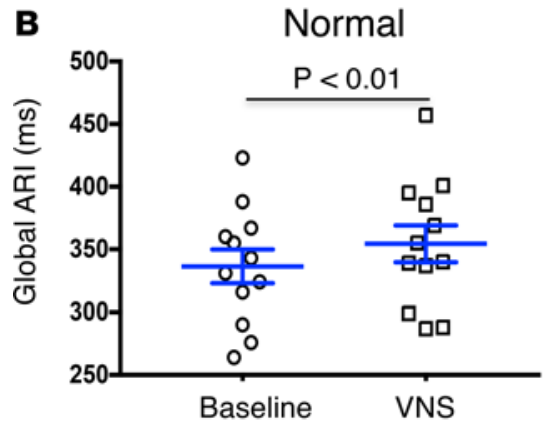

C

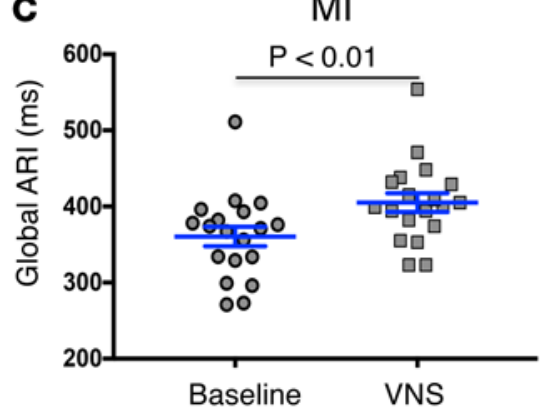

D

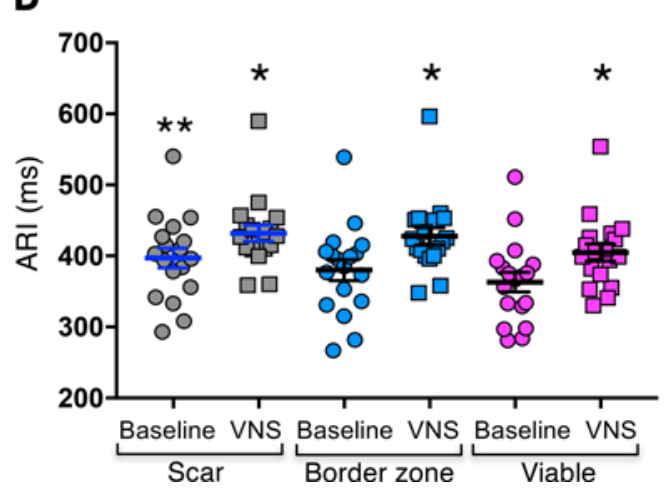

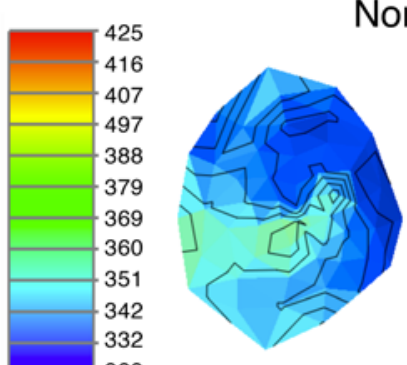

Baseline

\section{Normal}

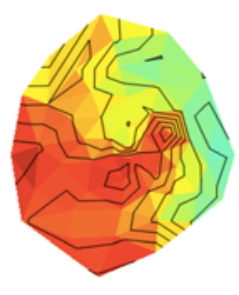

VNS ARI (ms)
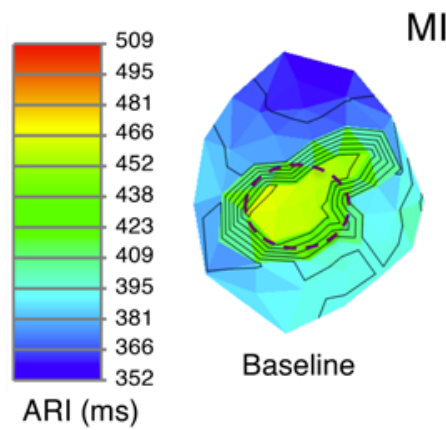

MI
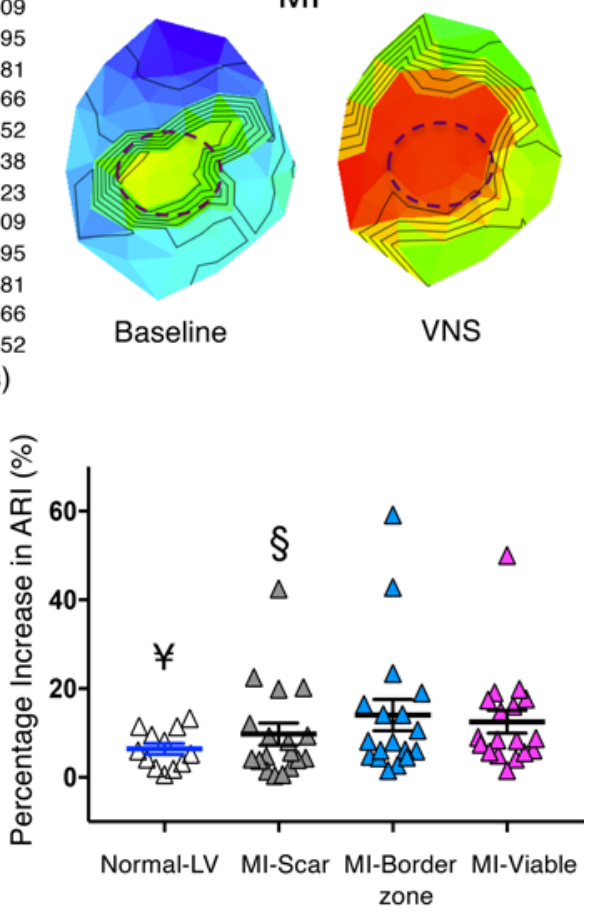

Figure 5. Electrophysiological response to VNS. (A) Examples of unipolar electrograms obtained from the sock electrode and used for ARI analysis from normal hearts and from viable, border zone, and scar regions of infarcted hearts are shown. (B) Global ARI (mean ARI from all 56 electrodes) in normal animals ( $n=12$ animals) increased with VNS (left panel, $P<0.01$, Wilcoxon signed rank test). Example of a polar map (right panel) from 1 normal animal shows that increases were observed in all regions. (C) The global ARI in infarcted hearts ( $n=18$ animals) also significantly increased with VNS (left panel, $P<0.01$, Wilcoxon signed rank test). Example of polar maps obtained from 1 infarcted animal is shown (right panel). In infarcted hearts, VNS has a significant impact on regional ARIs, particularly noticeable along peri-infarct regions (location of scar is marked with dashed lines on the polar maps of this animal). (D) Scar regions, at baseline, demonstrated the greatest mean ARI ( ${ }^{*} P<0.001$, linear mixed effects model, $n=18$ infarcted animals). In addition, all regions, even scar, demonstrate a significant increase in mean ARI from baseline ( ${ }^{*} P<0.01$, linear mixed effects model, $n=18$ infarcted animals). Furthermore, when the percentage increase in ARI with VNS is compared across various regions of infarcted hearts, consistent with the ACh findings, scar shows the least response compared with viable and border zone regions ( ${ }^{\$} P<0.01$, linear mixed effects model, $n=18$ infarcted animals). However, as with ACh results, there was no statistical difference in the response to VNS of scar compared with normal hearts $\left({ }^{*} P=0.1\right.$, linear mixed effects model, $n=18$ infarcted animals). ARI, activation recovery interval; MI, myocardial infarction; VNS, vagal nerve stimulation; LV, left ventricle.

with ischemic cardiomyopathy and previous characterization of this porcine model (16-19). In addition, in the 5 infarcted hearts from which samples of scar, border zone, and normal myocardium were obtained, islands of viable myocardium were evident within fibrotic areas designated as scar, likely giving rise to the electrical recordings observed in these regions (Figure 7). 
A

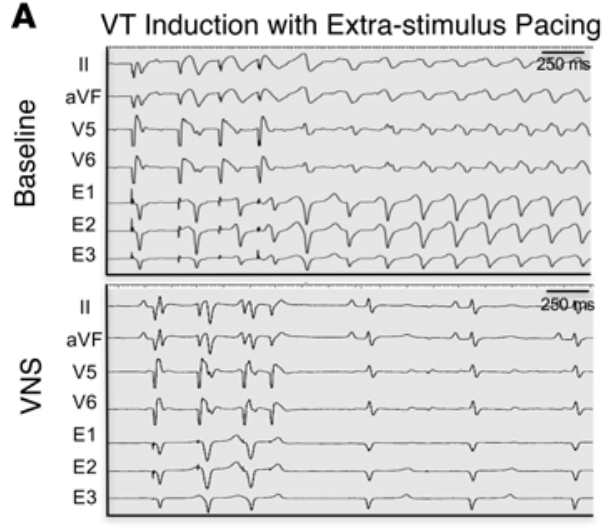

C

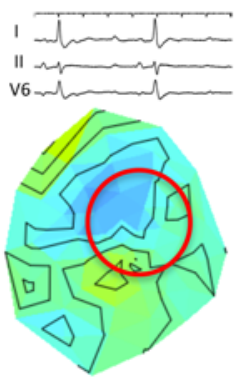

Sinus Rhythm

$\begin{array}{llllllllllll}78 & 71 & 64 & 57 & 51 & 44 & 37 & 30 & 24 & 16 & 10 & 3.0\end{array}$

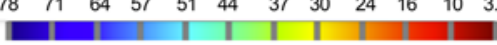

Activation time (ms)

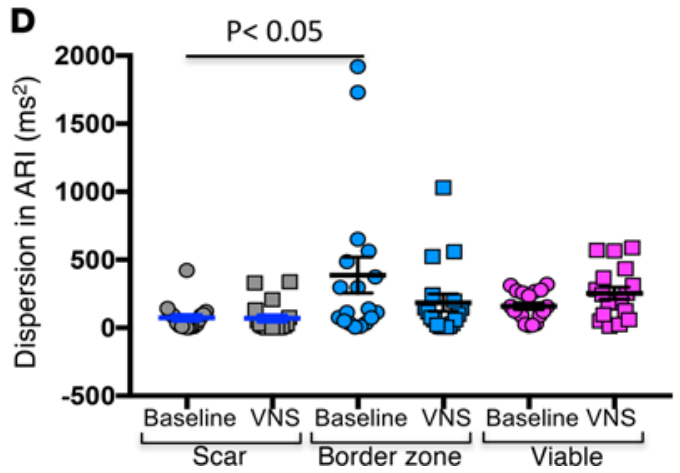

B
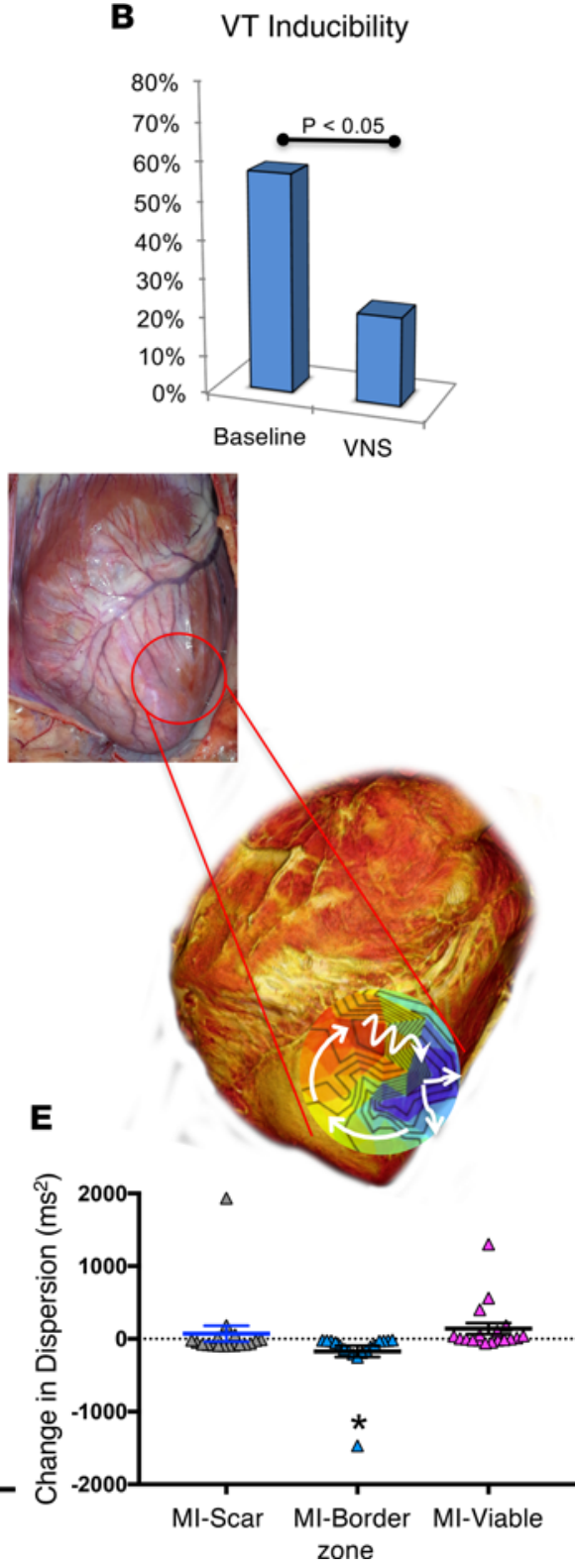

Figure 6. Arrhythmia inducibility and dispersion of repolarization. (A) An example of VT/VF induction using extrastimulus pacing from the RV endocardium is shown. Prior to VNS, this infarcted animal was inducible for VT, which degenerated into $V F$ and required defibrillation. During intermittent VNS, VT/VF was no longer inducible with triple extrastimuli, and effective refractory period was reached. (B) Intermittent VNS significantly reduced the proportion of animals that were inducible for VT ( $n=$ 21 infarcted, $P=0.04$, McNemar's test). (C) Epicardial activation map during VT in an infarcted animal that was inducible at baseline shows significant area of slowed conduction (multiple contours, zig-zag arrow) with "early" activation meeting "late activation" along the anterolateral border zone, likely contributing to reentry. (D) The dispersion in ARI at baseline and during VNS is shown across scar, border zone, and viable regions in infarcted hearts. Regional analysis showed that the dispersion in ARI in border zone regions was greater than scar regions $(P<0.05$, linear mixed effects model, $n=18$ infarcted animals) at baseline. (E) Intermittent VNS significantly reduced the dispersion in ARI of border zone regions compared with scar and viable areas $\left({ }^{*} P<0.01\right.$, linear mixed effects model, $n=18$ infarcted animals). ARI, activation recovery interval; MI, myocardial infarction; VNS, vagal nerve stimulation; VT, ventricular tachyarrhythmia.

\section{Discussion}

MI causes diminished central parasympathetic drive, as evidenced by direct neuronal recordings in vivo and neurotransmitter measurements in infarcted and normal hearts. We show for the first time to our knowledge that augmenting this drive with VNS reduces VT inducibility, even acutely, in chronic infarcted hearts. Furthermore, a mechanism for the antiarrhythmic benefit of VNS is electrical stabilization of infarct border zones, with reduced regional excitability and decreased heterogeneity in action potential duration. Acute electrical stabilization by VNS likely takes advantage of preserved cardiac ACh levels and an intact parasympathetic cardiac neuronal network.

In this study, ACh levels in infarcted hearts were preserved 6-8 weeks after MI, while the input - particularly from the left vagal trunk - to the parasympathetic neurons in the VIV ganglia was reduced. Although the total proportion of convergent and efferent parasympathetic neurons - as well as the primary relationships of these neurons - remained intact, there was increasing sympathetic input to convergent parasympathetic neurons, providing evidence for increased sympathetic drive coupled with lack of parasympathetic drive. Importantly, restoring central drive via VNS acutely reduced VT inducibility in the setting of chronic infarction, a finding that has important implications for patients with cardiomyopathy and recurrent VT and electrical storm. Finally, a mechanism for 


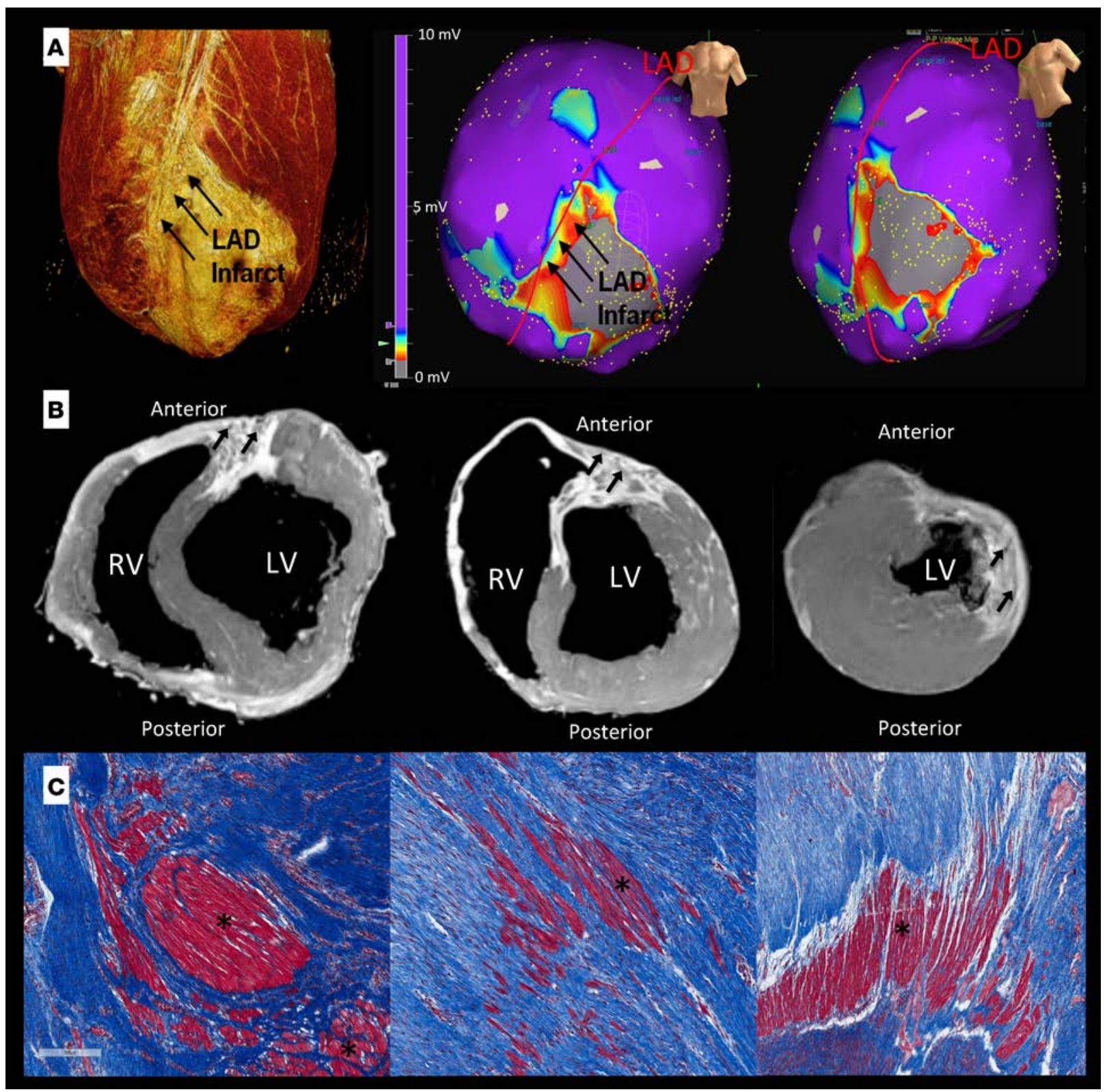

Figure 7. Delineation of scar via MRI, electroanatomic mapping, and histology. (A) Location and extent of scar on epicardial rendering of cardiac MRI correlated well with electroanatomic mapping. Black arrows indicate course of the left anterior descending coronary artery. (B) Multiple high-resolution ex vivo cardiac MRI demonstrated areas of viable myocardium within "scar" regions. Areas with delayed enhancement (scar) are often heterogenous. Images shown are from 3 different infarcted hearts. Arrows indicate regions of viable myocardium within regions of delayed enhancement. (C) Histological examination using Masson's Trichrome staining demonstrated viable islands of myocardium in areas demarcated as "scar" based on voltage criteria on electroanatomic mapping. Original magnification, 10x. Asterisk delineates viable islands of myocardium. LAD, left anterior descending coronary artery; LV, left ventricle; RV, right ventricle.

the acute benefit of VNS is suggested by the decrease in dispersion of repolarization of border zone regions, reducing risk of reentry.

Mechanisms of parasympathetic dysfunction. The mechanisms behind parasympathetic dysfunction and increased risk of sudden cardiac death in patients with cardiomyopathy are not clear. In patients with heart failure, abnormal heart rate variability and baroreceptor sensitivity exist and increase risk of sudden death (3-6), but it is unknown which factors (cardiac neurotransmitters, thoracic and cardiac ganglia neural remodeling, or the CNS) are the primary drivers of parasympathetic dysfunction. The vago-sympathetic trunk, the primary highway of the parasympathetic nervous system, carries preganglionic cardiomotor fibers from the brainstem that synapse on postganglionic neurons within the intrinsic cardiac ganglia on 
the surface of the heart. The cardiac ganglia are an important processing center for the heart, responding to beat-by-beat changes in mechanoelectrical function $(14,20,21)$. Parasympathetic neurons within these ganglia send postganglionic projections to atrial and ventricular myocardium, which release ACh. Unlike NE levels - which were clearly reduced in scar and border zone regions, confirming previous studies demonstrating lack of sympathetic response in certain scar areas and abnormal remodeling of sympathetic nerves along border zones regions (22-24) - ACh levels in infarcted hearts remained predominantly intact. In particular, there was no reduction in ACh levels along border zone regions. This data suggested that ACh is available for release, if central parasympathetic drive is restored.

Cardiac ganglia neurons of infarcted hearts respond abnormally to cardiac interventions, such as a decrease in preload (15). In this study, basal activity of parasympathetic neurons in the VIV ganglia was altered by MI. In particular, alterations in activity of neurons receiving left vagal input were observed. This laterality may be due to the ganglia used for recordings or location of the infarct (which primarily involved the LV). Multiple ganglia innervate the ventricular myocardium, and it is possible that if neural activity had been recorded from the dorsal ventricular fat pad, abnormal responses to right VNS may have been noted. Given the infarct location, it's also possible that afferent signaling to the brainstem - especially the left nucleus tractus solitarius, which provides input to the left nucleus ambiguus and left dorsal motor nucleus of the vagus - is altered and leads to a reduction of parasympathetic drive through the left vagal trunk (25-27). However, both vagal trunks are needed to maintain normal parasympathetic tone, and transection of one trunk can have significant electrophysiological consequences in porcine and canine hearts $(28,29)$. Therefore, decreased parasympathetic outflow through the left vagus can have profound effects on the ventricular myocardium, particularly in the infarcted region.

An evaluation of the type of parasympathetic neurons within the cardiac ganglia revealed that the total number of convergent parasympathetic neurons was unchanged between normal and infarcted hearts, and the primary underlying relationships of these neurons remained intact. However, the proportion of convergent neurons that would normally receive only parasympathetic input decreased, and the proportion receiving sympathetic input increased. These findings provide important insight into the neural remodeling that occurs with MI, with evidence for sympathetic activation and central parasympathetic withdrawal at the level of the cardiac ganglia. Furthermore, these observations begin to explain the beneficial effects of VNS in the setting of chronic MI, as increasing central parasympathetic drive can utilize the intact, but perhaps underused, pathways to provide the necessary clutch on sympathetic activation at the level of the cardiac ganglia.

Given that ACh levels in infarcted hearts remained unchanged, we hypothesized that VNS may be able to suppress electrophysiological instability or heterogeneity in ventricular repolarization that leads to ventricular arrhythmias, reducing inducibility of VT. Low to moderate levels of VNS can reduce ventricular arrhythmias due to myocardial ischemia and can, therefore, decrease mortality acutely ( 8 , $10,30-32$ ), and termination of VT has been observed in case reports of patients with recurrent arrhythmia after administration of phenylephrine to increase vagal tone (33). However, institution of VNS just prior to occurrence of ischemia is not feasible in patients. In this study, mild to moderate levels of VNS reduced inducibility of VT weeks after MI. The mechanism for this reduction could not have been due to the previously described effects of chronic VNS, such as connexin-43 expression or reduction of inflammation, as these would take time to develop. In this study, acute release of ACh likely led to cardiac electrical stability in infarcted hearts.

Many ventricular arrhythmias in infarcted hearts are due to changes in conduction velocity and differences in regional action potential duration, setting up the substrate for reentry (34-38). Scars contain islands of live myocardium, but border zone regions demonstrated the greatest heterogeneity in ARI, as these regions often contain a complex intertwining of variable amounts of viable myocardium and fibrotic tissue $(16,19,39,40)$. Intermittent VNS significantly reduced ARI dispersion in these regions. This acute electrical stabilization of infarct border zones provides an insight into the antiarrhythmic mechanisms of VNS and has important implications for patients with ischemic cardiomyopathy who suffer from refractory VT and defibrillator shock weeks to years after the initial insult. Approximately $30 \%$ of patients with cardiomyopathy and defibrillator shocks continue to experience recurrent arrhythmias despite medical therapy and catheter ablation procedures, presenting a dilemma for clinical electrophysiologists $(11-13,41)$. VNS represents a potential therapeutic option that can be used far after occurrence of MI and can acutely reduce burden of these arrhythmias. 
Surprisingly, the increase in global ARI of infarcted hearts was greater than in normal hearts. Given the decrease in central drive, this increase in response may be due to upreguation of muscarinic receptors in the heart. Although an increase in receptor density has not been evaluated in ischemic cardiomyopathy, in a guinea pig model of pacing-induced heart failure, M2 receptors have been shown to be upregulated, and VNS is able to normalize these receptor levels (42).

Myocardial neurotransmitter levels. Decreased electrical response to VNS in scar regions compared with border zone and viable myocardium is not surprising, given that $\mathrm{MI}$ is known to damage nerve fibers within the scar regions. The reduction in nerve fibers and myocytes would explain both the decrease in ACh levels and the decreased electrophysiological response to VNS in scar compared with border zone and viable regions of infarcted hearts. However, it was surprising that, even in the scar regions, ACh levels and response to VNS, although reduced, were not statistically different from similar regions in normal hearts. It's important to note that areas of low voltage typically classified as "scar" represent a complex intermingling of fibrosis and islands of viable myocardium, also observed in this study $(19,39)$. Therefore, the response to VNS seen in scar regions is likely due to viable islands of myocardium and neurites within scars. These axons have intact cell bodies in the cardiac ganglia located in the basal regions of the heart that can repair injured fibers. The fact that NE levels in scar regions were reduced compared with normal hearts while ACh levels remained predominantly intact suggests that sympathetic reinnervation is affected by a different processes than cholinergic innervation. Post-ganglionic sympathetic fibers originate from neurons in the sympathetic chain, which is located farther from the heart, and their reinnervation may be hindered by different molecules (43) than cholinergic fibers, whose cell bodies are anatomically closer. Also, cholinergic transdifferentiation likely plays an important role in the preserved ACh levels observed (44).

Limitations. We classified scars as low-voltage areas with voltage less than $0.5 \mathrm{mV}$ that still demonstrated a distinct bipolar recording, as this definition has been validated in clinical electrophysiological studies and these sites are used as targets for ablation. Therefore, it is possible that extremely dense scars with no clear voltage recording may not contain significant ACh levels or respond to VNS. However, these types of sites were rarely encountered in our infarct model and, when observed, often demonstrated unclear $\mathrm{T}$ waves and were excluded. Epicardial unipolar electrograms were recorded in this study. Therefore, intramural and endocardial effects were not assessed. ARIs were not corrected for heart rate, which was modest, as any changes in dispersion seen with VNS that may be due to bradycardia are still physiologically and clinically relevant. Fixed pacing during VNS was not performed, as pacing is known to alter autonomic tone and cause sympathetic activation $(45,46)$. Furthermore, we have previously shown that ARIs during VNS correlate most strongly with inotropy (or $d P / d t$ ), even more than heart rate (47).

\section{Methods}

Creation of myocardial infarcts. Yorkshire pigs (S\&S Farms) were sedated (tiletamine-zolazepam, 4-8 m/ $\mathrm{kg}$ ), intubated, and placed under anesthesia (isoflurane, 1\%-5\%). A guide sheath (Boston Scientific) was introduced from the right femoral artery and used to cannulate the left coronary artery under fluoroscopic guidance. Microspheres (5-7 ml, Polysciences) were injected through a luminal angioplasty balloon (FoxCross, Abbot) that was advanced into the mid-left anterior descending artery and inflated (Supplemental Figure 1; ref. 17). T wave inversions and ST segment elevations/depressions were noted on the surface ECG. External cardioversion was performed if the animal developed sustained ventricular arrhythmias.

Surgical preparation. Six to 8 weeks after MI, Yorkshire pigs were sedated (tilteamine-zolazepam, 4-8 $\mathrm{mg} / \mathrm{kg}$ ), intubated, and placed under general anesthesia (isoflurane, 1\%-5\%). A 12-lead ECG was obtained via CardioLab System (GE Healthcare). Systemic arterial blood pressure was continuously monitored via a femoral arterial sheath. Arterial blood gas levels were measured hourly. After completion of surgical exposure, anesthetics were switched from isoflurane to $\alpha$-chloralose (Sigma-Aldrich; $50 \mathrm{mg} / \mathrm{kg}$ initial bolus, then $20-35 \mathrm{mg} / \mathrm{kg} / \mathrm{hr})(14,28,48)$ for in vivo neuronal recordings, followed by stabilization period of 1 hour. Animals were euthanized by an overdose of i.v. sodium pentobarbital (Med-Pharmex Inc.; $100 \mathrm{mg} /$ $\mathrm{kg}$ ) followed by saturated KCL (Sigma-Aldrich; $1-2 \mathrm{mg} / \mathrm{kg}$ ).

Neurotransmitter measurements. Bipolar epicardial voltage mapping (NAVX, St. Jude Medical) was performed in all infarcted hearts 6-8 weeks after MI using multielectrode catheters (2-2-2 duodecapolar catheters, St. Jude Medical). Based on the voltage map, areas of scar (voltage $0.05-0.5 \mathrm{mV}$ ), border zone (voltage $0.5-1.5 \mathrm{mV}$ ), and viable (voltage $>1.5 \mathrm{mV}$ ), along with any late or fractionated electrograms, were marked. 
These values have been previously validated in the clinical setting to delineate scar, border zone, and viable regions during catheter ablation procedures $(49,50)$. Border zone and scar regions are characterized by low-voltage electrograms that often have prolonged duration or fractionation (Figure 2A) and represent targets of catheter ablation in patients (51). Samples (3-mm punch-biopsies) from the LV anterior wall were obtained from 15 normal animals and from scar, border zone, and viable regions in 14 infarcted animals. All animals were measured 6-8 weeks after MI. Neurotransmitter content was also measured across the LV apex, anterior, and lateral walls on the epicardium in 12 normal hearts (to evaluate regional changes that may be present and given that, in infarcted hearts, these were the predominant regions affected by scar). Finally, ACh and NE content from paired epicardial vs. endocardial samples was also assessed in 9 normal hearts. Epicardial to endocardial comparisons were made by obtaining samples directly across from each other in the heart. For multiple samples from similar regions of a heart, the weighted average of the neurotransmitter content for that animal was calculated and used for analysis.

Immediately after biopsy in vivo, tissue was placed in liquid nitrogen and stored at $-80^{\circ} \mathrm{C}$. For neurotransmitter analysis, tissue was pulverized and aliquots homogenized in $300 \mu 1$ of $0.1 \mathrm{M}$ perchloric acid containing deuterated ACh (acetylcholine-1,1,2,2 $\mathrm{d}_{4}$ chloride; $0.5 \mu \mathrm{M}, \mathrm{CDN}$ Isotopes). A standard curve for ACh (Sigma-Aldrich) was made up in the above homogenization buffer with final concentrations in the range of $0-500 \mathrm{nM}$. Homogenates were centrifuged and filtered $(0.22-\mu \mathrm{m}$ filters) before analysis by HPLC-MS. Samples were chromatographically separated (Scherzo SS-C18 column, $3 \mu \mathrm{m}$, Imtakt) using 0\%-60\% ammonium formate gradient and acetonitrile (100 mM ammonium formate/ acetonitrile; $65 / 35$ ) in $0.5 \%$ formic acid. ACh was quantified by a linear ion trap mass spectrometer (Applied Biosystems MDS SCIEX 4000 QTrap mass spectrometer) as previously described (52).

For NE analysis, aliquots $(100 \mu \mathrm{l})$ were centrifuged, and catecholamines were purified by alumina extraction. NE standards in the above homogenization buffer were analyzed by HPLC, with electrochemical detection in parallel with the tissue samples. Catecholamines were chromatographically separated (Microsorb C18 column, Agilent Technologies) using a mobile phase consisting of $360 \mathrm{mg} / 1$ sodium octane sulfonate (Sigma-Aldrich) and $6 \%(\mathrm{v} / \mathrm{v})$ acetonitrile in $75 \mathrm{mM}$ sodium phosphate buffer ( $\mathrm{pH} 3.0)$. NE was detected and quantified by coulometric detector (Coulochem III, ESA) (53).

In vivo neuronal recordings. VIV ganglia were accessed at the fat pad the atrioventricular junction, beneath the left atrial appendage (Supplemental Figure 1). In 10 infarcted and 15 normal animals, a linear microelectrode array (16 platinum/iridium electrodes, $500 \mu \mathrm{m}$ spacing, impedance $0.3-0.5 \mathrm{M} \Omega$ at $1 \mathrm{kHz}$, MicroProbes) was placed in the VIV fat pad. Electrode wires, as well as ground and reference electrodes, were connected to a 16-channel microelectrode amplifier with a head-stage preamplifier (A-M Systems Inc., model 3600). Neuronal waveforms (filter $300 \mathrm{~Hz}$ to $3 \mathrm{KHz}$ ) were recorded continuously via a data acquisition system (Cambridge Electronic Design). Data analysis and signal processing was performed offline using the software Spike2 (Cambridge Electronic Design) (14).

Characterization of parasympathetic neurons. To determine which neurons receive parasympathetic input, bipolar spiral cuff electrodes (Cyberonics) were placed around the bilateral cervical vagi after lateral neck cutdown (Supplemental Figure 1B). Right and left VNS were performed in a random fashion. Any increase or decrease in firing activity of recorded neurons during stimulation compared with prestimulation (baseline) was quantified and used to identify parasympathetic neurons. VNS threshold was defined as the current that led to a $10 \%$ decrease in heart rate $(1 \mathrm{~ms}, 20 \mathrm{~Hz})$. Right and left VNS were each performed in random order for 1 minute at $1 \mathrm{~Hz}$ at this current to avoid hemodynamic changes that may reflexively alter neuronal activity, and to measure neural activity independent of cardiac function. Basal activity of neurons that responded to right and/or left VNS was analyzed for the minute prior to stimulation. Firing frequency (basal activity) of these neurons in the minute prior to VNS was compared in infarcted and normal animals.

Once a neuron was classified as receiving parasympathetic input, it was then further classified as either purely efferent or convergent based on its response to afferent stimuli (14). Activity of neurons were assessed in the minute before afferent stimulation (baseline) and compared during stimulation in 13 of 15 normal and 9 of 10 infarcted animals. Neurons responding to right and/or left VNS and at least one afferent stimulus were defined as convergent, and those that only responded to right and/or left VNS were defined as purely efferent (14). Afferent stimuli consisted of mechanical stimuli (gentle touch) applied for 10 seconds at the RV outflow tract, RV apex, LV mid-anterior wall, and LV apex and occlusion of the IVC and aorta for 30 seconds using a snare occluder. At least 5 minutes was allowed in between interventions to allow for neural activity to return to baseline/stabilize. 
To evaluate whether these parasympathetic convergent or efferent neurons also received sympathetic input, each neuron's response to right and left stellate stimulation was assessed. The right and left stellate ganglia were isolated behind the parietal pleura. Bipolar needle electrodes attached to a photoelectric isolation unit and Grass stimulator (Grass Technologies, S88 and PSIU6) were used for stimulation (54). The current required for a $10 \%$ increase in heart rate or systolic blood pressure at $4 \mathrm{~Hz}$ and $4 \mathrm{~ms}$ was defined as threshold. Then - to avoid hemodynamic effects of stimulation, which could activate neural reflexes - stimulation was performed for 1 minute at $1 \mathrm{~Hz}$, consistent with other published data for assessment of neural response $(14,15)$.

Electrophysiological study. In 21 infarcted and 12 normal animals, unipolar epicardial electrograms were continuously obtained using a 56-electrode sock placed over the ventricles, in vivo (Prucka Cardio Lab System, GE Healthcare). The location of each electrode was recorded and color coded (Supplemental Figure 1C). In addition, in infarcted hearts, bipolar voltage electroanatomic mapping was performed as described above, and location of each electrode overlying scar, border zone, or viable myocardium meticulously was noted. ARI was analyzed from unipolar electrograms of each electrode using iScaldyn (University of Utah, Salt Lake City, Utah, USA) $(47,54,55)$. Briefly, activation time was defined from the origin to the minimum $d V / d t$ of the activation wavefront of the unipolar electrogram, and recovery time was defined as the start of activation to the maximum $d V / d t$ of repolarization wavefront. ARI was calculated by subtracting activation time from repolarization time. ARI has been previously validated and shown to correlate well with local action potential duration (56-59). In addition, it serves as a method of recording action potential duration without causing myocardial injury. In this manuscript, anterior refers to the ventral aspect and posterior refers to the dorsal aspect of the animal. Regional ARIs (scar, viable, border zone) were quantified in 18 of 21 hearts.

Cardiac magnetic resonance imaging with delayed enhancement was obtained in 16 infarcted hearts ex vivo after completion of ARI recordings and VT inducibility testing. Gadolinium was infused 20 minutes prior to euthanasia, and the heart was explanted immediately after euthanasia. MR images were used to confirm the location and extent of the scar (Supplemental Figure 1D). In addition, to confirm the presence of scar in low-voltage areas, samples from scar, border zone, and viable regions from 5 infarcted hearts were obtained prior to MRI and fixed in formalin overnight. Sections $(5 \mu \mathrm{m})$ of each region were stained with Masson's Trichrome to evaluate for fibrosis.

In normal animals, regional ARIs from LV apex, anterior, lateral, and right ventricular anterior wall were used for comparison to similar regions in infarcted hearts. In the 12 normal animals, the median number of electrodes used to compare mean ARIs in each region of normal hearts was 4 (range of 3-6). For the 18 infarcted animals, the median number of electrodes used for analysis of dispersion was 6 (range 4-7) in the scar region, 6 (range 4-10) in the border zone region, and 6 in the viable region around the scar (range 4-8). To qualitatively evaluate regional ARIs, polar maps were created from the sock electrode using an electrode template (Supplemental Figure 1).

Intermittent bilateral VNS (10 Hz, 1 ms, 15 seconds "on", 15 seconds "off") was performed via bipolar electrodes around the cervical vagi. The current required to cause a $10 \%$ decrease in heart rate was defined as threshold, and stimulation was performed at $1.2 \times$ this current. ARIs and hemodynamic variables were analyzed before and during VNS. After 1 minute of intermittent VNS, during which ARI and hemodynamic data was obtained in sinus rhythm, VT inducibility was evaluated. The duration of intermittent VNS ranged between 3 and 5 minutes, depending on VT inducibility and the number of extrastimuli required to induce VT.

VT inducibility was assessed before and during VNS using programmed ventricular stimulation (Micropace, EPS320) at two different cycle lengths, up to three extrastimuli (down to $200 \mathrm{~ms}$ or effective refractory period), from two different sites (right ventricular endocardium and LV anterior epicardium). If VT was induced from one specific site at baseline, this same site was used to induce VT during VNS. If sustained VT was inducible at baseline, a minimum wait period of 30 minutes was allowed after cardioversion before VNS and repeat inducibility testing.

Conditional probability analysis. Conditional probability analysis $(14,15)$ was used to evaluate the likelihood that a neuron classified as parasympathetic based on its response to right or left VNS responded to another stimulus such as inferior vena cava (IVC) occlusion. The potential for a connection between stimulus $\mathrm{X}$ and $\mathrm{Y}$ within neurons characterized as VNS responsive was measured in each animal as the conditional probability that a parasympathetic neuron that responded to stimulus $Y$ also responded to stimulus X. Conditional probability was estimated as the number of neurons that responded to both stimulus $\mathrm{X}$ 
and $\mathrm{Y}$, divided by the number of neurons that responded only to $\mathrm{X}$, and was quantified in a matrix format. Conditional probabilities that were greater than or equal to 0.6 were illustrated in a graphical network to assess overall connectivity of parasympathetic neurons in the cardiac ganglia $(14,15)$.

Statistics. Data are reported as mean \pm SEM. For comparison of mean and regional ACh, NE, and ARI, as well as ARI dispersion, linear mixed effects models were used and corrected for multiple comparisons for statistical significance using the Benjamini-Hochberg procedure to control the FDR at 5\%. Dispersion in ARI was defined as the variance in mean ARIs. For electrical response to VNS, percent changes from baseline were calculated first, prior to comparing various regions. Paired two-tailed Student's $t$ test was used to compare changes from baseline for a specific region to VNS and for hemodynamic response to VNS. Unpaired one-tailed Student's $t$ test was used to assess differences in stimulation threshold and basal neural activity in infarcted and normal animals. For evaluation of response to afferent and sympathetic stimuli, first, neuronal activity was compared at baseline vs. during stimuli and then, a statistical test, developed for cortical neurons based on the Skellam distribution, was used (60). This test has also been previously validated for intrinsic cardiac neurons (14). A $\chi^{2}$ test was used to compare the proportion of the type of neurons (e.g., convergent and receiving sympathetic input) in infarcted to normal hearts. Comparison of VT inducibility before and during VNS was performed using McNemar's test. $P<0.05$ was considered statistically significant.

Study approval. Approval for all animal procedures was obtained from the UCLA Chancellor's Animal Research Committee. All animal procedures were performed according to the IACUC guidelines and the NIH Guide for the Care and Use of Laboratory Animals (National Academies Press, 2011).

\section{Author contributions}

MV was involved in design of the study, conducting all experiments, acquiring data, analyzing electrophysiological data, and writing the manuscript. SS was involved in conducting neural recording experiments and analyzing neural data. PSR assisted in conducting the in vivo neural recording experiments and analyzing data. DY was involved in conducting the electrophysiological in vivo experiments and acquiring data. WRW was involved in analyzing neurotransmitter data and writing the manuscript. DH was involved in creating myocardial infarcts, conducting the neural recording experiments, and analyzing data. KY was involved in conducting the electrophysiological experiments and acquiring data. TI was involved in creating myocardial infarcts, conducting the electrophysiological experiments, and acquiring ARI data. BAH was involved analyzing and interpreting the neurotransmitter data and writing the manuscript. KS was involved in design of study and writing the manuscript.

\section{Acknowledgments}

This study was supported by NIH 1DP2HL132356, AHA National Fellow to Faculty Transition Award 11FTF7550004 to MV, and NIH1OT2OD023848.

Address correspondence to: Marmar Vaseghi, UCLA Cardiac Arrhythmia Center \& Neurocardiology Research Center of Excellence, 100 Medical Plaza, Suite 660, Los Angeles, California 90095, USA. Phone: 310.206.2235; Email: mvaseghi@mednet.ucla.edu.

1. Vaseghi M, Shivkumar K. The role of the autonomic nervous system in sudden cardiac death. Prog Cardiovasc Dis. 2008;50(6):404-419

2. Zipes DP, Rubart M. Neural modulation of cardiac arrhythmias and sudden cardiac death. Heart Rhythm. 2006;3(1):108-113.

3. Farrell TG, et al. Risk stratification for arrhythmic events in postinfarction patients based on heart rate variability, ambulatory electrocardiographic variables and the signal-averaged electrocardiogram. J Am Coll Cardiol. 1991;18(3):687-697.

4. Farrell TG, et al. Baroreflex sensitivity and electrophysiological correlates in patients after acute myocardial infarction. Circula tion. 1991;83(3):945-952.

5. Kleiger RE, Miller JP, Bigger JT, Moss AJ. Decreased heart rate variability and its association with increased mortality after acute myocardial infarction. Am J Cardiol. 1987;59(4):256-262.

6. La Rovere MT, Bigger JT, Marcus FI, Mortara A, Schwartz PJ. Baroreflex sensitivity and heart-rate variability in prediction of total cardiac mortality after myocardial infarction. ATRAMI (Autonomic Tone and Reflexes After Myocardial Infarction) Investigators. Lancet. 1998;351(9101):478-484.

7. Ando M, et al. Efferent vagal nerve stimulation protects heart against ischemia-induced arrhythmias by preserving connexin 43 protein. Circulation. 2005;112(2):164-170.

8. Kent KM, Smith ER, Redwood DR, Epstein SE. Electrical stability of acutely ischemic myocardium. Influences of heart rate 
and vagal stimulation. Circulation. 1973;47(2):291-298.

9. Kolman BS, Verrier RL, Lown B. The effect of vagus nerve stimulation upon vulnerability of the canine ventricle: role of sympathetic-parasympathetic interactions. Circulation. 1975;52(4):578-585.

10. Rosenshtraukh L, et al. Mechanisms for vagal modulation of ventricular repolarization and of coronary occlusion-induced lethal arrhythmias in cats. Circ Res. 1994;75(4):722-732.

11. Dukkipati SR, et al. Long-term outcomes of combined epicardial and endocardial ablation of monomorphic ventricular tachycardia related to hypertrophic cardiomyopathy. Circ Arrhythm Electrophysiol. 2011;4(2):185-194.

12. Kuck KH, et al. Catheter ablation of stable ventricular tachycardia before defibrillator implantation in patients with coronary heart disease (VTACH): a multicentre randomised controlled trial. Lancet. 2010;375(9708):31-40.

13. Kumar S, et al. Long-term outcomes after catheter ablation of ventricular tachycardia in patients with and without structural heart disease. Heart Rhythm. 2016;13(10):1957-1963.

14. Beaumont E, et al. Network interactions within the canine intrinsic cardiac nervous system: implications for reflex control of regional cardiac function. J Physiol (Lond). 2013;591(18):4515-4533.

15. Rajendran PS, et al. Myocardial infarction induces structural and functional remodelling of the intrinsic cardiac nervous system. J Physiol (Lond). 2016;594(2):321-341.

16. Nakahara S, et al. Distribution of late potentials within infarct scars assessed by ultra high-density mapping. Heart Rhythm. 2010;7(12):1817-1824.

17. Nakahara S, et al. Characterization of myocardial scars: electrophysiological imaging correlates in a porcine infarct model. Heart Rhythm. 2011;8(7):1060-1067.

18. Reddy VY, Wrobleski D, Houghtaling C, Josephson ME, Ruskin JN. Combined epicardial and endocardial electroanatomic mapping in a porcine model of healed myocardial infarction. Circulation. 2003;107(25):3236-3242.

19. Rutherford SL, Trew ML, Sands GB, LeGrice IJ, Smaill BH. High-resolution 3-dimensional reconstruction of the infarct border zone: impact of structural remodeling on electrical activation. Circ Res. 2012;111(3):301-311.

20. Armour JA. Potential clinical relevance of the 'little brain' on the mammalian heart. Exp Physiol. 2008;93(2):165-176.

21. Huang MH, Sylvén C, Pelleg A, Smith FM, Armour JA. Modulation of in situ canine intrinsic cardiac neuronal activity by locally applied adenosine, ATP, or analogues. Am J Physiol. 1993;265(4 Pt 2):R914-R922.

22. Ajijola OA, et al. Focal myocardial infarction induces global remodeling of cardiac sympathetic innervation: neural remodeling in a spatial context. Am J Physiol Heart Circ Physiol. 2013;305(7):H1031-H1040.

23. Barber MJ, Mueller TM, Henry DP, Felten SY, Zipes DP. Transmural myocardial infarction in the dog produces sympathectomy in noninfarcted myocardium. Circulation. 1983;67(4):787-796.

24. Cao JM, et al. Relationship between regional cardiac hyperinnervation and ventricular arrhythmia. Circulation. 2000;101(16):1960-1969.

25. Janig W. The Integrative Action of the Autonomic Nervous System: Neurobiology of Homeostasis. Cambridge, England: Cambridge University Press; 2006:13-34.

26. Norgren R. Projections from the nucleus of the solitary tract in the rat. Neuroscience. 1978;3(2):207-218.

27. Standish A, Enquist LW, Schwaber JS. Innervation of the heart and its central medullary origin defined by viral tracing. Science. 1994;263(5144):232-234.

28. Yamakawa K, et al. Vagal nerve stimulation activates vagal afferent fibers that reduce cardiac efferent parasympathetic effects Am J Physiol Heart Circ Physiol. 2015;309(9):H1579-H1590.

29. Ardell JL, Rajendran PS, Nier HA, KenKnight BH, Armour JA. Central-peripheral neural network interactions evoked by vagus nerve stimulation: functional consequences on control of cardiac function. Am J Physiol Heart Circ Physiol. 2015;309(10):H1740-H1752.

30. Myers RW, et al. Beneficial effects of vagal stimulation and bradycardia during experimental acute myocardial ischemia. Circulation. 1974;49(5):943-947.

31. Yoon MS, Han J, Tse WW, Rogers R. Effects of vagal stimulation, atropine, and propranolol on fibrillation threshold of normal and ischemic ventricles. Am Heart J. 1977;93(1):60-65.

32. Zuanetti G, De Ferrari GM, Priori SG, Schwartz PJ. Protective effect of vagal stimulation on reperfusion arrhythmias in cats. Circ Res. 1987;61(3):429-435.

33. Waxman MB, Wald RW. Termination of ventricular tachycardia by an increase in cardiac vagal drive. Circulation. 1977;56(3):385-391.

34. Allessie MA, Bonke FI, Schopman FJ. Circus movement in rabbit atrial muscle as a mechanism of tachycardia. II. The role of nonuniform recovery of excitability in the occurrence of unidirectional block, as studied with multiple microelectrodes. Circ Res. 1976;39(2):168-177.

35. Baker LC, London B, Choi BR, Koren G, Salama G. Enhanced dispersion of repolarization and refractoriness in transgenic mouse hearts promotes reentrant ventricular tachycardia. Circ Res. 2000;86(4):396-407.

36. Kuo CS, Munakata K, Reddy CP, Surawicz B. Characteristics and possible mechanism of ventricular arrhythmia dependent on the dispersion of action potential durations. Circulation. 1983;67(6):1356-1367.

37. Wit AL, Allessie MA, Bonke FI, Lammers W, Smeets J, Fenoglio JJ. Electrophysiologic mapping to determine the mechanism of experimental ventricular tachycardia initiated by premature impulses. Experimental approach and initial results demonstrating reentrant excitation. Am J Cardiol. 1982;49(1):166-185.

38. de Bakker JM, et al. Slow conduction in the infarcted human heart. 'Zigzag' course of activation. Circulation. 1993;88(3):915-926.

39. Nakahara S, et al. Characterization of the arrhythmogenic substrate in ischemic and nonischemic cardiomyopathy implications for catheter ablation of hemodynamically unstable ventricular tachycardia. J Am Coll Cardiol. 2010;55(21):2355-2365.

40. Watanabe A, Seki A, Fishbein MC, Shivkumar K, Vaseghi M. Arrhythmogenic Right Ventricular Cardiomyopathy: Electroarchitecture of the Substrate. HeartRhythm Case Rep. 2016;2(1):47-51.

41. Mathuria N, Tung R, Shivkumar K. Advances in ablation of ventricular tachycardia in nonischemic cardiomyopathy. Curr Car diol Rep. 2012;14(5):577-583. 
42. DeMazumder D, Kass DA, O'Rourke B, Tomaselli GF. Cardiac resynchronization therapy restores sympathovagal balance in the failing heart by differential remodeling of cholinergic signaling. Circ Res. 2015;116(10):1691-1699.

43. Gardner RT, et al. Targeting protein tyrosine phosphatase $\sigma$ after myocardial infarction restores cardiac sympathetic innervation and prevents arrhythmias. Nat Commun. 2015;6:6235.

44. Kanazawa $\mathrm{H}$, et al. Heart failure causes cholinergic transdifferentiation of cardiac sympathetic nerves via gp130-signaling cytokines in rodents. J Clin Invest. 2010;120(2):408-421.

45. Herre JM, Thames MD. Responses of sympathetic nerves to programmed ventricular stimulation. J Am Coll Cardiol. 1987;9(1):147-153.

46. Taylor JA, Morillo CA, Eckberg DL, Ellenbogen KA. Higher sympathetic nerve activity during ventricular (VVI) than during dual-chamber (DDD) pacing. J Am Coll Cardiol. 1996;28(7):1753-1758.

47. Yamakawa K, et al. Electrophysiological effects of right and left vagal nerve stimulation on the ventricular myocardium. Am $J$ Physiol Heart Circ Physiol. 2014;307(5):H722-H731.

48. Beam DM, Neto-Neves EM, Stubblefield WB, Alves NJ, Tune JD, Kline JA. Comparison of isoflurane and $\alpha$-chloralose in an anesthetized swine model of acute pulmonary embolism producing right ventricular dysfunction. Comp Med. 2015;65(1):54-61.

49. Cesario DA, et al. Value of high-density endocardial and epicardial mapping for catheter ablation of hemodynamically unstable ventricular tachycardia. Heart Rhythm. 2006;3(1):1-10.

50. Marchlinski FE, Callans DJ, Gottlieb CD, Zado E. Linear ablation lesions for control of unmappable ventricular tachycardia in patients with ischemic and nonischemic cardiomyopathy. Circulation. 2000;101(11):1288-1296.

51. Harada T, Stevenson WG, Kocovic DZ, Friedman PL. Catheter ablation of ventricular tachycardia after myocardial infarction: relation of endocardial sinus rhythm late potentials to the reentry circuit. J Am Coll Cardiol. 1997;30(4):1015-1023.

52. Hasan W, Woodward WR, Habecker BA. Altered atrial neurotransmitter release in transgenic p75(-/-) and gp130 KO mice. Neurosci Lett. 2012;529(1):55-59.

53. Habecker BA, et al. Regulation of cardiac innervation and function via the p 75 neurotrophin receptor. Auton Neurosci. 2008;140(1-2):40-48.

54. Yagishita D, et al. Sympathetic nerve stimulation, not circulating norepinephrine, modulates T-peak to T-end interval by increasing global dispersion of repolarization. Circ Arrhythm Electrophysiol. 2015;8(1):174-185.

55. Vaseghi M, Lux RL, Mahajan A, Shivkumar K. Sympathetic stimulation increases dispersion of repolarization in humans with myocardial infarction. Am J Physiol Heart Circ Physiol. 2012;302(9):H1838-H1846.

56. Haws CW, Lux RL. Correlation between in vivo transmembrane action potential durations and activation-recovery intervals from electrograms. Effects of interventions that alter repolarization time. Circulation. 1990;81(1):281-288.

57. Millar CK, Kralios FA, Lux RL. Correlation between refractory periods and activation-recovery intervals from electrograms: effects of rate and adrenergic interventions. Circulation. 1985;72(6):1372-1379.

58. Chinushi $\mathrm{M}$, et al. Correlation between the effective refractory period and activation-recovery interval calculated from the intracardiac unipolar electrogram of humans with and without dl-sotalol treatment. Jpn Circ J. 2001;65(8):702-706.

59. Yue AM, Paisey JR, Robinson S, Betts TR, Roberts PR, Morgan JM. Determination of human ventricular repolarization by noncontact mapping: validation with monophasic action potential recordings. Circulation. 2004;110(11):1343-1350.

60. Shin HC, Aggarwal V, Acharya S, Schieber MH, Thakor NV. Neural decoding of finger movements using Skellam-based maximum-likelihood decoding. IEEE Trans Biomed Eng. 2010;57(3):754-760. 